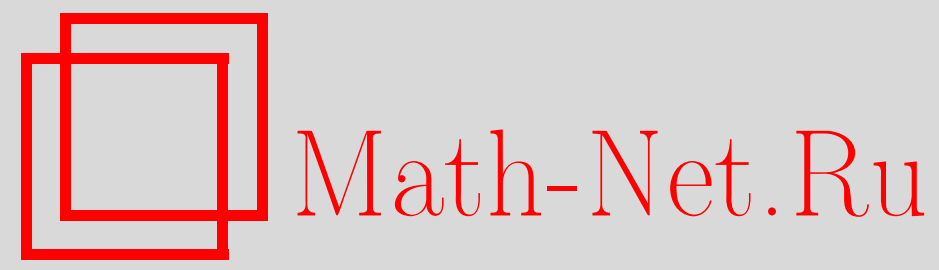

А. Б. Пиуновский, Управляемая скачкообразная модель с дисконтированием при наличии ограничений, Теория вероятн. и ее примен., 1997, том 42, выпуск 1, 108-133

DOI: https://doi.org/10.4213/tvp1715

Использование Общероссийского математического портала Math-Net.Ru подразумевает, что вы прочитали и согласны с пользовательским соглашением http://www. mathnet.ru/rus/agreement

Параметры загрузки:

IP: 34.239 .49 .27

26 апреля 2023 г., 14:03:54

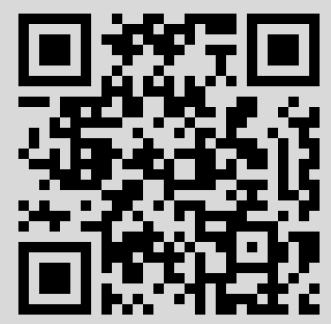




\title{
УПРАВЛЯЕМАЯ СКАЧКООБРАЗНАЯ МОДЕЛЬ С ДИСКОНТИРОВАНИЕМ ПРИ НАЛИЧИИ ОГРАНИЧЕНИЙ ${ }^{1)}$
}

\begin{abstract}
Рассматривается задача, в которой заданные функционалы от траекторий должны удовлетворять системе неравенств. Как обычно, требуется построить стратегию управления, доставляющую экстремальное значение критерия.

В статье исследовано пространство стратегических мер, получены необходимые и достаточные условия оптимальности, разработан алгоритм построения оптимальной стратегии для марковского случая, приведен точно решенный содержательный пример.

Ключевые слова и фразы: оптимальное управление, скачкообразные случайные процессы, функциональные ограничения, выпуклое программирование, теория массового обслуживания.
\end{abstract}

\section{1. Введение}

Математическая теория управляемых скачкообразных случайных процессов складывалась в 70-х годах [1]-[3] и получила дальнейшее развитие в работах многих авторов [4]-[8]. Чаще всего априорно ограничнваются простейшими стратегиями - селекторами [1]-[4], [6]-[8]; рандомизированные стратегии рассматривались в [5]. При заданном начальном распределении вероятностей всякая стратегия однозначно определяет стохастическую меру на траекториях, конструктивное ее построение содержится в [1], [4]. Ставится задача поиска стратегии управления, доставляющей экстремальное значение критерия; под критерием, как правило, понимают математическое ожидание некоторого действительного функционала на траекториях (интегрального типа) [1], [2], [4], [7], [8]. Весьма распространен частный случай однородных моделей с дисконтированием [2], [8].

Один из главных вопросов, возникающих при исследовании задач управления, - каким классом стратегий можно ограничиться при поиске решения. Как правило, для однородных дисконтированных мар-

*Институт физико-технических проблем, ул. Пречистенка, 13/7, 119034 Москва, Россия.

1) Работа выполнена при поддержке Российского фонда фундаментальных исследований, грант № 95-01-00191. 
ковских моделей доказывается достаточность стационарных марковских селекторов; влияние начального распределения на оптимальную стратегию встречается достаточно редко.

Принципиально иная ситуация возникает при исследовании задач управления с ограничениями. Такая постановка задачи для управляемых однородных марковских последовательностей приведена в [9]. Некоторые новые результаты, в том числе для немарковских моделей, получены в [10]-[13]. Основной результат заключается в том, что при поиске решения достаточно ограничиться классом смесей $N+1$ стационарного селектора, где $N$ - число ограничений. Скачкообразные модели с ограничениями ранее, по-видимому, не рассматривались, за исключением статьи [14], где приведено исследование модели с конечным горизонтом при очень кратких доказательствах. Диффузионные управляемые модели с ограничениями изучались в [15].

Настоящая работа посвящена моделям с дисконтированием. Исследования ведутся в пространстве стратегических мер с использованием общих теорем выпуклого программирования. В разделе 3 доказана выпуклость указанного пространства и изучена структура его крайних точек. В разделе 4 продемонстрировано использование метода множителей Лагранжа для решения исходной задачи; в разделе 5 изложен алгоритм построения решения для марковского случая. Разрешимость задачи для марковской модели с конечным пространством состояний установлена в разделе 6 , там же доказано, что решение можно найти в виде смеси $N+1$ стационарного марковского селектора.

Традиционную область приложений теории управляемых скачкообразных процессов представляют модели массового обслуживания [3], $[16],[17]$. В разделе 7 приведен точно решенный пример оптимизации системы массового обслуживания при одном ограничении. Показано, что оптимальная стратегия не может быть стационарным селектором и существенно зависит от начального распределения.

\section{2. Определения, постановка задачи и вспомогательные результаты}

Построение управляемой модели проводится по аналогии с [5]. Пусть заданы произвольные борелевские пространства $(X, \mathcal{B}(X))$ - пространство состояний, и $(A, \mathcal{B}(A))$ - пространство управлений, в которых зафиксируем метризуемые топологии $\tau^{X}, \tau^{A}$ такие, что минимальная $\sigma$-алгебра, содержащая $\tau^{X}\left(\tau^{A}\right)$, совпадает с $\mathcal{B}(X)(B(A))$. Присоединим к пространствам $X, A$ изолированные точки $x_{\infty}, a_{\infty}$ и положим $X_{\infty} \triangleq X \cup\left\{x_{\infty}\right\}, A_{\infty} \triangleq A \cup\left\{a_{\infty}\right\}$ с соответствующей модификацией $\sigma$-алгебр. В дальнейшем используются обозначения $\mathbf{R}_{+} \triangleq(0, \infty), \mathbf{R}_{+}^{0} \triangleq$ $[0, \infty)$. Положим $\left(\Omega^{0}, \mathcal{F}^{0}\right) \triangleq\left(\left(X \times \mathbf{R}_{+}\right)^{\infty}, \mathcal{B}\left(\left(X \times \mathbf{R}_{+}\right)^{\infty}\right)\right)$ и присоединим к $\Omega^{0}$ все последовательности вида $\left(x_{0}, \theta_{1}, x_{1}, \ldots, \theta_{n-1}, x_{n-1}, \infty, x_{\infty}, \infty\right.$, 
$\left.x_{\infty}, \ldots\right), n \geqslant 1$, где $(\forall k=1, \ldots, n-1) \theta_{k} \neq \infty, x_{k} \neq x_{\infty}$. После соответствующей модификации $\sigma$-алгебры $\mathcal{F}^{0}$ получаем основное измеримое пространство $(\Omega, \mathcal{F})$. Положим $T_{0} \triangleq 0, T_{n} \triangleq \theta_{1}+\theta_{2}+\cdots+\theta_{n}$; $T_{\infty} \triangleq \lim _{n \rightarrow \infty} T_{n}$

$$
\xi_{t} \triangleq \sum_{n \geqslant 0} I\left\{T_{n} \leqslant t<T_{n+1}\right\} x_{n}+I\left\{T_{\infty} \leqslant t\right\} x_{\infty} .
$$

Здесь и ниже $I-$ функция-индикатор.

Введем целочисленную случайную меру

$$
\mu(\omega, d t, d x)=\sum_{n \geqslant 1} I\left\{T_{n}<\infty\right\} \delta_{\left(T_{n}, x_{n}\right)}(d t, d x)
$$

(где $\delta_{y}(\cdot)$ - мера Дирака, сосредоточенная в точке $y$ ), а также непрерывное справа семейство $\sigma$-алгебр $\left\{\mathcal{F}_{t}\right\}_{t \geqslant 0}: \mathcal{F}_{t} \triangleq \sigma\left\{x_{0}, \mu\left([0, \tau] \times \Gamma^{X}\right), \tau \in[0, t]\right.$, $\left.\Gamma^{X} \in \mathcal{B}(X)\right\} ; \mathcal{F}_{\infty} \triangleq \bigvee_{t \geqslant 0} \mathcal{F}_{t}$. Пусть $\mathcal{P}$ есть $\sigma$-алгебра предсказуемых множеств на $\Omega \times \mathbf{R}_{+}^{0}$, связанная с $\left\{\mathcal{F}_{t}\right\}_{t \geqslant 0}[18]$. Очевидно, скачкообразный процесс $\xi_{t}$ согласован и непрерывен справа, а следовательно, прогрессивно измерим [6].

Предположим, что задана конечная мера интенсивности скачков $\lambda\left(\Gamma^{X} \mid a, \omega, t\right), \mathcal{B}\left(A_{\infty}\right) \times \mathcal{P}$-измеримая по $(a, \omega, t)$ при произвольном фиксированном $\Gamma^{X} \in \mathcal{B}(X) ; \lambda\left(\left\{\xi_{t-}(\omega)\right\} \mid a, \omega, t\right)=0$. Интенсивность выхода $\lambda(X \mid a, \omega, t) \leqslant K<\infty$ считаем равномерно ограниченной; кроме того, считаем $\lambda(X \mid a, \omega, t)=0$ при $t \geqslant T_{\infty}$.

Наиболее хорошо изучены марковские модели, в которых $\lambda\left(\Gamma^{X}\right.$ । $a, \omega, t)=\tilde{\lambda}\left(\Gamma^{X} \mid a, \xi_{t-}(\omega), t\right)$; функции $\tilde{\lambda}\left(\Gamma^{X} \mid a, x, t\right)$ заданы и измеримы при всех $\Gamma^{X} \in \mathcal{B}(X)$. В дальнейшем, когда речь пойдет о марковских моделях, тильда опускается.

$\mathrm{O}$ п р е д е л е н и е. Стратегией $\pi$ называется $\mathcal{P}$-измеримая переходная вероятность $\pi(\cdot \mid \omega, t)$ на $\left(A_{\infty}, \mathcal{B}\left(A_{\infty}\right)\right)$, причем $\pi\left(a_{\infty} \mid \omega, t\right)=$ $I\left\{T_{\infty} \leqslant t\right\}$. Если существует такой предсказуемый процесс $\varphi(\omega, t)$ со значениями в $A_{\infty}$, что $\pi\left(\Gamma^{A} \mid \omega, t\right)=I\left\{\Gamma^{A} \ni \varphi(\omega, t)\right\}$, то стратегия называется селектором и обозначается символом $\varphi$.

Важный класс стратегий образуют марковские селекторы вида $\varphi\left(t, \xi_{t-}(\omega)\right)$, где $\varphi: \mathbf{R}_{+}^{0} \times X_{\infty} \rightarrow A_{\infty}$ - измеримое отображение такое, что $\varphi^{-1}\left(a_{\infty}\right)=\left\{\left(t, x_{\infty}\right), t \in \mathbf{R}_{+}^{0}\right\}$. Поскольку процесс $\xi_{t-}(\omega)$ предсказуем, отображение $\varphi\left(t, \xi_{t-}(\omega)\right) \mathcal{P}$-измеримо и поэтому действительно задает селектор. Марковский селектор называется стационарным, если он не зависит от времени: $\varphi(t, x)=\varphi(x)$.

Очевидно, для любой стратегии $\pi$ случайная мера

$$
\nu^{\pi}(\omega, d t, d x) \triangleq\left[\int_{A} \pi(d a \mid \omega, t) \lambda(d x \mid a, \omega, t)\right] d t
$$


предсказуема. Кроме того, $\nu^{\pi}(\omega,\{t\} \times X)=\nu^{\pi}\left(\omega,\left[T_{\infty}, \infty\right) \times X\right)=0$. Поэтому для всякой стратегии $\pi$ и для любого начального распределения $P_{0}$ на $X$ существует единственная вероятностная мера $P_{P_{0}}^{\pi}$ на $(\Omega, \mathcal{F})$ такая, что $P_{P_{0}}^{\pi}\left\{x_{0} \in \Gamma^{X}\right\}=P_{0}\left(\Gamma^{X}\right)$ при всех $\Gamma^{X} \in \mathcal{B}(X)$ и $\nu^{\pi}$ является предсказуемой проекцией меры $\mu[5],[19]$. При фиксированных $P_{0}$ и $\pi$ $\sigma$-алгебры $\mathcal{F}_{t}, \mathcal{F}$ пополняются множествами нулевой меры $P_{P_{0}}^{\pi}$. Мера $P_{P_{0}}^{\pi}$ на $(\Omega, \mathcal{F})$ строится в явном виде следуюшим образом. Как известно [19], $\forall \Gamma^{X} \in \mathcal{B}(X)$ предсказуемая функция

$$
\Lambda\left(\Gamma^{X} \mid \omega, t\right) \triangleq \int_{A} \pi(d a \mid \omega, t) \lambda\left(\Gamma^{X} \mid a, \omega, t\right)
$$

имеет вид

$$
\begin{aligned}
\Lambda\left(\Gamma^{X} \mid \omega, t\right)= & I\{t=0\} \Lambda\left(\Gamma^{X} \mid \omega, 0\right) \\
& +\sum_{0 \leqslant n<\infty} I\left\{T_{n}<t \leqslant T_{n+1}\right\} \Lambda^{n}\left(\Gamma^{X} \mid \omega, t-T_{n}\right),
\end{aligned}
$$

где $\Lambda^{n}\left(\Gamma^{X} \mid \omega, \tilde{t}\right)$ - набор $\left(\mathcal{F}_{T_{n}} \times \mathcal{B}\left(\mathbf{R}_{+}\right)\right)$-измеримых функций. Функции $\Lambda^{n}(\cdot \mid \omega, \tilde{t})$ при фиксированных $\omega, \tilde{t}$ являются мерами на $(X, \mathcal{B}(X))$. Пусть $\left.\left.\widehat{H}_{n} \triangleq X \times(] 0, \infty\right] \times X_{\infty}\right)^{n}, n=1,2, \ldots$ Мера $P_{0}$ на $\widehat{H}_{0}$ задана. Предположим, что построена мера $P_{P_{0}}^{\pi}$ на $\widehat{H}_{n}$, тогда мера $P_{P_{0}}^{\pi}$ на $\widehat{H}_{n+1}$ определяется формулами

$$
\begin{aligned}
& P_{P_{0}}^{\pi}\left(\Gamma^{\widehat{H}_{n}} \times(d \tilde{t}, d x)\right) \triangleq \int_{\Gamma^{\hat{H}_{n}}} I\left\{\theta_{n}<\infty\right\} P_{P_{0}}^{\pi}\left(d h_{n}\right) d \tilde{t} \\
& \times \Lambda^{n}\left(d x \mid h_{n}, \tilde{t}\right) \exp \left\{-\int_{0}^{\tilde{t}} \Lambda^{n}\left(X \mid h_{n}, s\right) d s\right\} \\
& P_{P_{0}}^{\pi}\left(\Gamma^{\widehat{H}_{n}} \times\left(\infty, x_{\infty}\right)\right) \triangleq \int_{\Gamma^{\hat{H}_{n}}} P_{P_{0}}^{\pi}\left(d h_{n}\right) \\
& \quad \times\left\{I\left\{\theta_{n}=\infty\right\}+I\left\{\theta_{n}<\infty\right\} \exp \left\{-\int_{0}^{\infty} \Lambda^{n}\left(X \mid h_{n}, s\right) d s\right\}\right\},
\end{aligned}
$$

где $\Gamma^{\widehat{H}_{n}} \in \boldsymbol{B}\left(\widehat{H}_{n}\right)$. Здесь и ниже $\Lambda^{n}\left(\Gamma^{X} \mid h_{n}, \tilde{t}\right)-$ функция, связанная с представлением (1) формулой $\Lambda^{n}\left(\Gamma^{X} \mid \omega, \tilde{t}\right)=\Lambda^{n}\left(\Gamma^{X} \mid h_{n}(\omega), \tilde{t}\right)$; $h_{n}(\omega)=\left(x_{0}, \theta_{1}, x_{1}, \ldots, \theta_{n}, x_{n}\right)$ - начальная часть последовательности $\omega \in \Omega$. Очевидно, при каждом $n \geqslant 0$ построенная мера сосредоточена на пространстве $n$-членных историй $\left\{h_{n}(\omega), \omega \in \Omega\right\}$. Согласно [22], существует единственная вероятностная мера $P_{P_{0}}^{\pi}$ на $(\Omega, \mathcal{F})$, проекции которой на пространства $n$-членных историй удовлетворяют равенствам (2).

Таким образом, полностью определен стохастический базис $(\Omega, \mathcal{F}$, $\left.P_{P_{0}}^{\pi},\left(\mathcal{F}_{t}\right)_{t \geqslant 0}\right)$, на котором задан скачкообразный процесс $\xi_{t}$. Начальное распределение в дальнейшем считается фиксированным, и индекс $P_{0}$ всюду опускается. Отметим, что из ограниченности интенсивности 
выхода вытекает, что $P^{\pi}\left\{T_{\infty}=\infty\right\}=1$. В дальнейштем $P_{t}^{\pi}-$ сужение $P^{\pi}$ на $\mathcal{F}_{t}$.

Пусть $(Y, \mathcal{Y})$ - измеримое пространство, $F(y)$ - действительная измеримая функция на $Y, F^{+} \triangleq F \vee 0, F^{-} \triangleq F \wedge 0$. Для всякой конечной меры $Q$ на $Y$ положим

$$
\int_{Y} F(y) Q(d y) \triangleq \int_{Y} F^{+}(y) Q(d y)+\int_{Y} F^{-}(y) Q(d y),
$$

где $\ll+\infty 》+\ll-\infty » \triangleq \ll+\infty »$. В дальнейшем полагаем, что действительные функции могут принимать значения $\pm \infty$.

Положим $(\widetilde{\Omega}, \tilde{\mathcal{F}}) \triangleq\left(\Omega \times \mathbf{R}_{+}^{0} \times A, \mathcal{F} \times \mathcal{B}\left(\mathbf{R}_{+}^{0}\right) \times \mathcal{B}(A)\right)$ и для каждой стратегии $\pi$ определим меру $Q^{\pi}$ на $\widetilde{\Omega}$ по формуле:

$$
Q^{\pi}(d \omega, d t, d a) \triangleq P^{\pi}(d \omega) e^{-\beta t} d t \pi(d a \mid \omega, t),
$$

где $\beta>0-$ фиксированный коэффициент дисконтирования. Символом $Q_{\mathcal{P}}^{\pi}$ обозначается сужение $Q^{\pi}$ на $\left(\Omega \times \mathbf{R}_{+}^{0} \times A, \mathcal{P} \times \mathcal{B}(A)\right)$. Если $F(\widetilde{\omega})-$ действительная измеримая функция на $\widetilde{\Omega}$ и $Q-$ конечная мера на $\widetilde{\Omega}$, то используется обозначение:

$$
\mathbf{F}(Q) \triangleq \int_{\tilde{\Omega}} F(\widetilde{\omega}) Q(d \tilde{\omega})
$$

Будем считать заданными конечное множество индексов $\mathcal{A}=$ $\{1,2, \ldots, N\}$ и действительные $\mathcal{P} \times \mathcal{B}(A)$-измеримые функции $R(\widetilde{\omega})$, $\left\{S_{\alpha}(\widetilde{\omega})\right\}_{\alpha \in \mathcal{A}}$ на $\widetilde{\Omega}$.

O п р е де ле н и е. Стратегии $\pi$, для которых выполнены неравенства

$$
\mathbf{S}_{\alpha}\left(Q^{\pi}\right) \leqslant 0, \quad \alpha \in \mathcal{A},
$$

называются допустимыми. Класс допустимых стратегий обозначается символом $\Pi$, и в дальнейшем предполагается, что $\Pi \neq \varnothing$.

Стратегии $\pi_{1}, \pi_{2}$ для которых $Q_{P}^{\pi_{1}}=Q_{P}^{\pi_{2}}$, считаются эквивалентными; в дальнейшем мы их не будем различать.

O п р е д е ле н и е. Стратегия $\pi^{*} \in \Pi$ называется оптимальной, если

$$
\mathbf{R}\left(Q^{\pi^{*}}\right)=\inf _{\pi \in \Pi} \mathbf{R}\left(Q^{\pi}\right) .
$$

Требуется построить оптимальную стратегию.

3 а м е ч а н и е. Функции $R, S_{\alpha}$ по смыслу являются функциями потерь (штрафов). Часто функции потерь задаются с помошью интенсивности $r(t, x, a)[1],[4],[5],[7]$, а минимизируемый функционал для селектора $\varphi$ имеет вид $\int_{\Omega} \int_{0}^{\infty} e^{-\beta t} r\left(t, \xi_{t-}(\omega), \varphi(\omega, t)\right) d t P^{\varphi}(d \omega)$. Очевидно, в этом случае $R(\omega, t, a)=r\left(t, \xi_{t-}(\omega), a\right)$. 
О п р е д е л е н и е. Меры $Q^{\pi}$ на $(\widetilde{\Omega}, \widetilde{\mathcal{F}})$, соответствующие различным стратегиям по формуле (3) (возможно, недопустимым), назовем стратегическими. Пространство стратегических мер обозначается символом $\widetilde{B}$. Положим $B \triangleq\left\{Q^{\pi} \in \widetilde{B}, \pi \in \Pi\right\}-$ пространство допустимых стратегических мер. Сужения $Q_{\mathcal{P}}^{\pi}$ образуют соответствующие пространства $\widetilde{B}_{\mathcal{P}}, B_{\mathcal{P}}$. Очевидно, множества $B \subseteq \widetilde{B}, B_{\mathcal{P}} \subseteq \widetilde{\boldsymbol{B}}_{\mathcal{P}}$ вложены в линейные пространства ограниченных зарядов на $(\widetilde{\Omega}, \widetilde{\mathcal{F}})$ и на $(\widetilde{\Omega}, \mathcal{P} \times$ $\mathcal{B}(A))$ соответственно, и имеет смысл ввести множества $\Phi, \widetilde{\Phi}, \Phi_{\mathcal{P}}, \widetilde{\Phi}_{\mathcal{P}}$ всех крайних точек в $B, \widetilde{B}, B_{\mathcal{P}}, \widetilde{B}_{\mathcal{P}}$ соответственно.

\section{3. Свойства пространств стратегических мер}

Лемма 1. Множество $\widetilde{B}$ всех стратеаических мер выпукло.

Д о к а 3 а т е л ь с т в о. Пусть $Q^{\pi_{1}}, Q^{\pi_{2}}-$ две стратегические меры; $\alpha^{\prime}+\alpha^{\prime \prime}=1, \alpha^{\prime}, \alpha^{\prime \prime}>0$. Нам нужно доказать, что $\alpha^{\prime} Q^{\pi_{1}}+\alpha^{\prime \prime} Q^{\pi_{2}} \in \widetilde{B}$.

Введем стохастическую меру $P \triangleq \alpha^{\prime} P^{\pi_{1}}+\alpha^{\prime \prime} P^{\pi_{2}}$ на $(\Omega, \mathcal{F}) ; \sigma$-алгебры $\mathcal{F}_{t}, \mathcal{F}$ пополняются множествами нулевой $P$-меры. Пусть $z^{\prime}(t, \omega)$, $z^{\prime \prime}(t, \omega)$ - процессы локальных плотностей $P^{\pi_{1}}, P^{\pi_{2}}$ относительно $P$ [18]. Так как $\alpha^{\prime} z^{\prime}+\alpha^{\prime \prime} z^{\prime \prime} \equiv 1$, можно рассмотреть стратегию $\pi \triangleq \alpha^{\prime} z_{-}^{\prime} \pi_{1}+$ $\alpha^{\prime \prime} z_{-}^{\prime \prime} \pi_{2}$. Согласно определению $P^{\pi}$, случайная мера $\alpha^{\prime} z_{-}^{\prime} \nu^{\pi_{1}}+\alpha^{\prime \prime} z_{-}^{\prime \prime} \nu^{\pi_{2}}$ является предсказуемой проекцией меры $\mu$ (для $P^{\pi}$ ); с другой стороны, эта же мера является предсказуемой проекцией $\mu$ для $P \triangleq \alpha^{\prime} P^{\pi_{1}}+\alpha^{\prime \prime} P^{\pi_{2}}$ [18], откуда следует, что $P^{\pi}=P=\alpha^{\prime} P^{\pi_{1}}+\alpha^{\prime \prime} P^{\pi_{2}}$. Теперь, используя известные свойства неотрицательных равномерно интегрируемых мартингалов $z^{\prime}, z^{\prime \prime}[18]$, легко убедиться, что для произвольной ограниченной неотрицательной измеримой функции $r(\widetilde{\omega})$ на $(\widetilde{\Omega}, \widetilde{\mathcal{F}})$ справедливо равенство

$$
\int_{\tilde{\Omega}} r(\widetilde{\omega}) Q^{\pi}(d \widetilde{\omega})=\alpha^{\prime} \int_{\tilde{\Omega}} r(\widetilde{\omega}) Q^{\pi_{1}}(d \widetilde{\omega})+\alpha^{\prime \prime} \int_{\widetilde{\Omega}} r(\widetilde{\omega}) Q^{\pi_{2}}(d \widetilde{\omega})
$$

т.e. $Q^{\pi}=\alpha^{\prime} Q^{\pi_{1}}+\alpha^{\prime \prime} Q^{\pi_{2}} \in \widetilde{B}$.

Следствие 1. Множества $\widetilde{B}_{\mathcal{P}}, B, B_{\mathcal{P}}$ выпуклы.

Доказательство очевидно.

Лемма 2. Если $Q_{\mathcal{P}} \in \widetilde{\Phi}_{\mathcal{P}}-$ крайняя точка $\widetilde{B}_{\mathcal{P}}$, то она порождаетсд некоторым селектором, т.е. $\exists \varphi: Q_{\mathcal{P}}^{\varphi}=Q_{\mathcal{P}}$.

Д о к а 3 а т е л ь с т в о. 1. Докажем вспомогательное утверждение. Предположим, что $\pi=\delta \pi_{1}+(1-\delta) \pi_{2}$, где $\pi_{1}$ и $\pi_{2}$ - некоторые стратегии, $\delta \in\left(0, \frac{1}{2}\right]-$ константа. Введем меру на $\left(\Omega \times \mathbf{R}_{+}^{0}, \mathcal{P}\right)$ :

$$
m(d \omega, d t) \triangleq p^{\pi}(d \omega) e^{-\beta t} d t,
$$

и предположим, что $\exists \widehat{\Gamma}^{A} \in \mathcal{B}(A), \exists \widehat{\Gamma}^{\Omega, t} \in \mathcal{P}: m\left(\widehat{\Gamma}^{\Omega, t}\right)>0$ и $\forall(\omega, t) \in \widehat{\Gamma}^{\Omega, t}$ $\pi_{1}\left(\widehat{\Gamma}^{A} \mid \omega, t\right) \neq \pi_{2}\left(\widehat{\Gamma}^{A} \mid \omega, t\right)$. Тогда точка $Q_{\mathcal{P}}^{\pi}$ не является крайней в $\widetilde{B}_{\mathcal{P}}$. 
Очевидно, что, не теряя общности, можно считать выполненными следуюшие соотношения: $\delta=\frac{1}{2}$;

$$
\begin{gathered}
\widehat{\Gamma}^{\Omega, t} \subseteq \rrbracket T_{n}, T_{n+1} \wedge M \rrbracket \text { при некоторых } n, M<\infty ; \\
\pi_{1}\left(\widehat{\Gamma}^{A} \mid \omega, t\right)>\pi_{2}\left(\widehat{\Gamma}^{A} \mid \omega, t\right)
\end{gathered}
$$

при всех $(\omega, t) \in \widehat{\Gamma}^{\Omega, t}$ и $\pi_{1}(\cdot \mid \omega, t)=\pi_{2}(\cdot \mid \omega, t)$ при всех $(\omega, t) \notin \widehat{\Gamma}^{\Omega, t}$. Теперь ясно, что меры $m_{1}$ и $m_{2}$, определенные по формуле (5) для стратегий $\pi_{1}$ и $\pi_{2}$, совпадают с мерой $m$ на множествах вида $\Gamma^{\Omega} \times \Gamma^{t}, \Gamma^{\Omega} \in \mathcal{F}_{T_{n}}$, $\Gamma^{t} \in \mathcal{B}\left(\mathbf{R}_{t}^{0}\right)$.

В дальнейшем $\Lambda^{n}\left(\Gamma^{X} \mid \omega, \tilde{t}\right)$ и $\Lambda_{1,2}^{n}\left(\Gamma^{X} \mid \omega, \tilde{t}\right)$ суть $\mathcal{F}_{T_{n}} \times \mathcal{B}\left(\mathbf{R}_{+}\right)$измеримые функции из представления (1) для функщий $\Lambda\left(\Gamma^{X} \mid \omega, t\right)$ и $\Lambda_{1,2}\left(\Gamma^{X} \mid \omega, t\right)$, заданных стратегиями $\pi$ и $\pi_{1,2}$. Отметим, что случай $\Lambda_{1}^{n} \equiv \Lambda_{2}^{n}$ не исключается.

Введем функции

$$
\widetilde{\gamma}^{1}(t) \triangleq \frac{\frac{1}{2}(d-1) e^{-t / 2}}{(d-1) e^{-t / 2}+1}, \quad \widetilde{\gamma}^{2}(t) \triangleq \frac{\frac{1}{2}(d+1) e^{-t / 2}}{(d+1) e^{-t / 2}-1}
$$

на отрезке $|t| \leqslant K M$, где $K-$ константа, ограничивагшая интенсивность выхода; $M-$ число из формулы $(6) ; d>2 e^{K M / 2}-1-$ произвольная фиксированная константа. Легко проверить, что справедливы соотношения:

$$
\begin{gathered}
0<\tilde{\gamma}^{1}(t)<\frac{1}{2}<\tilde{\gamma}^{2}(t)<1 \\
\frac{1}{2} \exp \left\{-\int_{0}^{t} \tilde{\gamma}^{1}(s) d s\right\}+\frac{1}{2} \exp \left\{-\int_{0}^{t} \tilde{\gamma}^{2}(s) d s\right\}=e^{-t / 2}, \\
\frac{1}{2}\left[\frac{1}{2}-\tilde{\gamma}^{1}(t)\right] \exp \left\{\int_{0}^{t}\left[\frac{1}{2}-\tilde{\gamma}^{1}(s)\right] d s\right\} \\
+\frac{1}{2}\left[\frac{1}{2}-\widetilde{\gamma}^{2}(t)\right] \exp \left\{\int_{0}^{t}\left[\frac{1}{2}-\widetilde{\gamma}^{2}(s)\right] d s\right\}=0
\end{gathered}
$$

при всех $t \in[-K M, K M]$. Так как

$$
\left|\int_{0}^{\tilde{t}}\left[\Lambda_{1}^{n}(X \mid \omega, s)-\Lambda_{2}^{n}(X \mid \omega, s)\right] d s\right| \leqslant K M
$$

при всех $\tilde{t} \geqslant 0$, то можно рассмотреть $\mathcal{F}_{T_{n}} \times \mathcal{B}\left(\mathbf{R}_{+}\right)$-измеримые функции

$$
\widehat{\gamma}^{1,2}(\omega, \tilde{t}) \triangleq \widetilde{\gamma}^{1,2}\left(\int_{0}^{\bar{t}}\left[\Lambda_{1}^{n}(X \mid \omega, s)-\Lambda_{2}^{n}(X \mid \omega, s)\right] d s\right),
$$

для которых, используя равенства (9), (10), легко установить тождества 
(при всех $\omega, \tilde{t}$ )

$$
\begin{aligned}
& \frac{1}{2} \exp \{-\int_{0}^{\bar{t}}\left[\left(1-\hat{\gamma}^{1}(\omega, s)\right) \Lambda_{2}^{n}(X \mid \omega, s)\right. \\
&\left.\left.+\widehat{\gamma}^{1}(\omega, s) \Lambda_{1}^{n}(X \mid \omega, s)\right] d s\right\} \\
&+ \frac{1}{2} \exp \left\{-\int_{0}^{\bar{t}}\left[\left(1-\widehat{\gamma}^{2}(\omega, s)\right) \Lambda_{2}^{n}(X \mid \omega, s)\right.\right. \\
&\left.\left.+\widehat{\gamma}^{2}(\omega, s) \Lambda_{1}^{n}(X \mid \omega, s)\right] d s\right\} \\
&= \exp \left\{-\int_{0}^{\bar{t}}\left[\frac{1}{2} \Lambda_{1}^{n}(X \mid \omega, s)+\frac{1}{2} \Lambda_{2}^{n}(X \mid \omega, s)\right] d s\right\} ; \\
& \frac{1}{2}\left[\frac{1}{2}-\widehat{\gamma}^{1}(\omega, \tilde{t})\right] \exp \left\{-\int_{0}^{\tilde{t}}\left[\left(1-\widehat{\gamma}^{1}(\omega, s)\right) \Lambda_{2}^{n}(X \mid \omega, s)\right.\right. \\
&\left.\left.\quad+\widehat{\gamma}^{1}(\omega, s) \Lambda_{1}^{n}(X \mid \omega, s)\right] d s\right\} \\
&+\frac{1}{2}\left[\frac{1}{2}-\widehat{\gamma}^{2}(\omega, \tilde{t})\right] \exp \left\{-\int_{0}^{\tilde{t}}\left[\left(1-\widehat{\gamma}^{2}(\omega, s)\right) \Lambda_{2}^{n}(X \mid \omega, s)\right.\right. \\
&\left.\left.\quad+\widehat{\gamma}^{2}(\omega, s) \Lambda_{1}^{n}(X \mid \omega, s)\right] d s\right\}=0 .
\end{aligned}
$$

Зафиксируем произвольное множество $\Gamma \in \mathcal{B}(X)$, умножим (11) на $\left[\frac{1}{2} \Lambda_{1}^{n}(\Gamma \mid \omega, \tilde{t})+\frac{1}{2} \Lambda_{2}^{n}(\Gamma \mid \omega, \tilde{t})\right]$ и сложим с тождеством (12), умноженным на $\left[\Lambda_{2}^{n}(\Gamma \mid \omega, \tilde{t})-\Lambda_{1}^{n}(\Gamma \mid \omega, \tilde{t})\right]$ :

$$
\begin{aligned}
\frac{1}{2}[(1 & \left.\left.-\widehat{\gamma}^{1}(\omega, \tilde{t})\right) \Lambda_{2}^{n}(\Gamma \mid \omega, \tilde{t})+\hat{\gamma}^{1}(\omega, \tilde{t}) \Lambda_{1}^{n}(\Gamma \mid \omega, \tilde{t})\right] \\
& \times \exp \left\{-\int_{0}^{\tilde{t}}\left[\left(1-\hat{\gamma}^{1}(\omega, s)\right) \Lambda_{2}^{n}(X \mid \omega, s)+\hat{\gamma}^{1}(\omega, s) \Lambda_{1}^{n}(X \mid \omega, s)\right] d s\right\} \\
& +\frac{1}{2}\left[\left(1-\widehat{\gamma}^{2}(\omega, \tilde{t})\right) \Lambda_{2}^{n}(\Gamma \mid \omega, \tilde{t})+\widehat{\gamma}^{2}(\omega, \tilde{t}) \Lambda_{1}^{n}(\Gamma \mid \omega, \tilde{t})\right] \\
& \times \exp \left\{-\int_{0}^{\tilde{t}}\left[\left(1-\hat{\gamma}^{2}(\omega, s)\right) \Lambda_{2}^{n}(X \mid \omega, s)+\hat{\gamma}^{2}(\omega, s) \Lambda_{1}^{n}(X \mid \omega, s)\right] d s\right\} \\
= & {\left[\frac{1}{2} \Lambda_{1}^{n}(\Gamma \mid \omega, \tilde{t})+\frac{1}{2} \Lambda_{2}^{n}(\Gamma \mid \omega, \tilde{t})\right] } \\
& \times \exp \left\{-\int_{0}^{\tilde{t}}\left[\frac{1}{2} \Lambda_{1}^{n}(X \mid \omega, s)+\frac{1}{2} \Lambda_{2}^{n}(X \mid \omega, s)\right] d s\right\} .
\end{aligned}
$$

Введем две новые стратегии $\pi_{1}^{\mathrm{H}}$ и $\pi_{2}^{\mathrm{H}}$ :

$$
\pi_{1,2}^{\mathrm{H}} \triangleq\left(1-\gamma^{1,2}\right) \pi_{2}+\gamma^{1,2} \pi_{1},
$$


rде

$$
\gamma^{1,2}(\omega, t) \triangleq \begin{cases}\widehat{\gamma}^{1,2}\left(\omega, t-T_{n}\right) & \text { при } t \in \rrbracket T_{n}, T_{n+1} \rrbracket \\ \frac{1}{2} & \text { при } t \notin \rrbracket T_{n}, T_{n+1} \rrbracket\end{cases}
$$

Tеперь равенство

$$
\begin{aligned}
\frac{1}{2} E^{\pi_{1}^{\mathrm{H}}} & {\left[\int_{0}^{\infty} \int_{A} \pi_{1}^{\mathrm{H}}(d a \mid \omega, t) r(\omega, t, a) e^{-\beta t} d t\right] } \\
& +\frac{1}{2} E^{\pi_{2}^{\mathrm{H}}}\left[\int_{0}^{\infty} \int_{A} \pi_{2}^{\mathrm{H}}(d a \mid \omega, t) r(\omega, t, a) e^{-\beta t} d t\right] \\
= & E^{\pi}\left[\int_{0}^{\infty} \int_{A} \pi(d a \mid \omega, t) r(\omega, t, a) e^{-\beta t} d t\right]
\end{aligned}
$$

для любой ограниченной $\mathcal{P} \times \mathcal{B}(A)$-измеримой функции $r(\widetilde{\omega})$ следует из (11), (12), (13) в соответствии с формулами (2) явного построения мер $P^{\pi}, P^{\pi_{1,2}^{\mathrm{H}}}$. Для функции $r(\omega, t, a) \triangleq I\left\{(\omega, t, a) \in \widehat{\Gamma}^{\Omega, t} \times \widehat{\Gamma}^{A}\right\}$ из соотношений $(7),(8)$ следует неравенство

$$
\int_{\tilde{\Omega}} r(\tilde{\omega}) Q_{\mathcal{P}}^{\pi_{1}^{\mathrm{H}}}(d \tilde{\omega})<\int_{\tilde{\Omega}} r(\tilde{\omega}) Q_{\mathcal{P}}^{\pi_{2}^{\mathrm{H}}}(d \tilde{\omega})
$$

Следовательно, точка $Q_{\mathcal{P}}^{\pi}$ не является крайней в $\widetilde{B}_{\mathcal{P}}$.

Можно заметить, что существуют процессы $z^{1}$ и $z^{2}$ локальных плотностей $P^{\pi_{1}^{\mathrm{H}}}$ и $P^{\pi_{2}^{\mathrm{H}}}$ относительно $P^{\pi}$, для которых выполнены соотношения

$$
\begin{gathered}
z^{1}(\omega, t)= \begin{cases}1, & \text { если }(\omega, t) \in \llbracket 0, T_{n} \rrbracket ; \\
\frac{1-2 \gamma^{2}(\omega, t)}{\gamma^{1}(\omega, t)-\gamma^{2}(\omega, t)}, & \text { если }(\omega, t) \in \rrbracket T_{n}, T_{n+1} \rrbracket ; \\
z^{1}\left(\omega, T_{n+1}\right), & \text { если }(\omega, t) \in \llbracket T_{n+1}, \infty \llbracket ;\end{cases} \\
z^{2}(\omega, t)= \begin{cases}1, & \text { если }(\omega, t) \in \llbracket 0, T_{n} \rrbracket ; \\
\frac{2 \gamma^{1}(\omega, t)-1}{\gamma^{1}(\omega, t)-\gamma^{2}(\omega, t)}, & \text { если }(\omega, t) \in \rrbracket T_{n}, T_{n+1} \llbracket ; \\
z^{2}\left(\omega, T_{n+1}\right), & \text { если }(\omega, t) \in \llbracket T_{n+1}, \infty \llbracket ;\end{cases} \\
z^{1,2}\left(\omega, T_{n+1}\right)=\left(\begin{array}{ll}
I\left\{T_{n+1}=\infty\right\}+I\left\{T_{n+1}<\infty\right\} \\
.
\end{array}\right. \\
. \exp \left\{-\int_{T_{n}}^{T_{n+1}}\left[\frac{1}{2}-\gamma^{1,2}(\omega, s)\right]\left[\Lambda_{2}(X \mid \omega, s)-\Lambda_{1}(X \mid \omega, s)\right] d s\right\} .
\end{gathered}
$$

Теперь $\pi=\frac{1}{2} z_{-}^{1} \pi_{1}^{\mathrm{H}}+\frac{1}{2} z_{-}^{2} \pi_{2}^{\mathrm{H}}$ и равенство $Q^{\pi}=\frac{1}{2} Q^{\pi_{1}^{\mathrm{H}}}+\frac{1}{2} Q^{\pi_{2}^{\mathrm{H}}}$ следует также из доказательства леммы 1. 
2. В дальнейшем $\pi$ - такая стратегия, что $Q_{\mathcal{P}}^{\pi}=Q_{\mathcal{P}}$. Используя I. 1 доказательства, нетрудно показать, что если $Q_{\mathcal{P}}^{\pi}-$ крайняя точка, то $\forall \Gamma^{A} \in \mathcal{B}(A) \forall \Gamma^{\Omega, t} \in \mathcal{P} \forall \delta \in(0,1)$ если $m\left(\Gamma^{\Omega, t}\right)>0$, то $\exists(\omega, t) \epsilon$ $\Gamma^{\Omega, t}: \pi\left(\Gamma^{A} \mid \omega, t\right)<\delta$, либо $\pi\left(\Gamma^{A} \mid \omega, t\right)>1-\delta$. Отсюда вытекает, что $\forall \Gamma^{A} \in \mathcal{B}(A) \pi\left(\Gamma^{A} \mid \omega, t\right)=1 \vee \pi\left(\Gamma^{A} \mid \omega, t\right)=0$ (m-п.н.). Более подробные выкладки аналогичны изложенным в [11].

3. Пусть $\rho$ - вполне ограниченная метризация $A ; \varepsilon_{k} \triangleq \frac{1}{2^{k}}(k=$ $1,2, \ldots) ;\left\{a_{1}^{k}, a_{2}^{k}, \ldots, a_{n_{k}}^{k}\right\}$ - такой набор точек из $A$, что

$$
A=\bigcup_{i=1}^{n_{k}} O\left(a_{i}^{k}, \varepsilon_{k}\right)
$$

где $O\left(a_{i}^{k}, \varepsilon_{k}\right) \triangleq\left\{a \in A: \rho\left(a, a_{i}^{k}\right)<\varepsilon_{k}\right\}$. Положим $G_{i}^{k} \triangleq\{(\omega, t) \in \Omega \times$ $\left.\mathbf{R}_{+}^{0}: \pi\left(O\left(a_{i}^{k}, \varepsilon_{k}\right) \mid \omega, t\right) \notin\{0,1\}\right\} \in \mathcal{P}$. Из пп. 1,2 доказательства следует, что $m\left(G_{i}^{k}\right)=0$, т.е. $m(G)=0$, где $G \triangleq \cup_{k, i} G_{i}^{k}$, поэтому достаточно построить искомое $\mathcal{P}$-измеримое отображение $\varphi$ из $\Omega \times \mathbf{R}_{+}^{0} \backslash G$ в $A$.

Зафиксируем произвольную точку $(\omega, t) \in \Omega \times \mathbf{R}_{+}^{0} \backslash G$. Пусть $\bar{O}^{1}-$ первая среди окрестностей $\left\{O\left(a_{i}^{1}, \varepsilon_{1}\right)\right\}_{i=1}^{n_{1}}$, для которой $\pi\left(\bar{O}^{1} \mid \omega, t\right)=1$. Аналогичным образом, $\bar{O}^{k+1} \triangleq \bar{O}^{k} \cap \hat{O}^{k+1}$, гце $\hat{O}^{k+1}-$ первая среди окрестностей $\left\{O\left(a_{i}^{k+1}, \varepsilon_{k+1}\right)\right\}_{i=1}^{n_{k+1}}$, для которой $\pi\left(\hat{O}^{k+1} \mid \omega, t\right)=1$. Ясно, что $\forall k$ справедливо равенство $\pi\left(\bar{O}^{k} \mid \omega, t\right)=1$, т.е. $\pi\left(\cap_{k \geqslant 1} \bar{O}^{k} \mid \omega, t\right)=$ 1 ; кроме того, очевидно, что множество $\bigcap_{k \geqslant 1} \bar{O}^{k}$ содержит ровно одну точку $a$, которую и принимаем за значение отображения $\varphi: \varphi(\omega, t)=a$. На множестве $G$ отображение $\varphi$ доопределяется произвольным образом.

Очевидно, из $\mathcal{P}$-измеримости стратегии $\pi$ вытекает предсказуемость процесса $\varphi$. Далее, нетрудно убедиться, что

$$
\begin{gathered}
\left\{\omega: \exists c: \int_{\mathbf{R}_{+}^{0}} \int_{X} c(t, x) e^{-\beta t} \nu^{\pi}(\omega, d t, d x)\right. \\
\left.\quad \neq \int_{\mathbf{R}_{+}^{0}} \int_{X} c(t, x) e^{-\beta t} \nu^{\varphi}(\omega, d t, d x)\right\} \\
\quad \subseteq\left\{\omega: \int_{\mathbf{R}_{+}^{0}} I\{(\omega, t) \in G\} e^{-\beta t} d t>0\right\} .
\end{gathered}
$$

Здесь $с$ пробегает класс непрерывных ограниченных функций на $\mathbf{R}_{+}^{0} \times X$. Из равенства $m(G)=0$ получаем, что $\nu^{\pi}=\nu^{\varphi}\left(P^{\pi}\right.$-п.н. $) \Longrightarrow P^{\pi}=P^{\varphi}[18]$. Теперь ясно, что $Q^{\pi}=Q^{\varphi}$ и $Q_{\mathcal{P}}^{\pi}=Q_{\mathcal{P}}^{\varphi}$.

Доказательство закончено. 


\section{4. Задача выпуклого программирования}

Ясно, что сформулированная в разделе 2 задача поиска оптимальной стратегии эквивалентна следующей задаче математического программирования в пространстве ограниченных зарядов на $(\widetilde{\Omega}, \widetilde{\mathcal{F}})$.

$\left.\begin{array}{l}\text { Найти точку, дающую инфимум функции } \mathbf{R}(Q) \text { на } \widetilde{B} \\ \text { при ограничениях } \mathbf{S}_{n}(Q) \leqslant 0, n=1,2, \ldots, N .\end{array}\right\}$

В дальнейшем будем говорить, что задача (14) неразрешима, если $B=$ $\left\{Q \in \widetilde{B}: \mathbf{S}_{n}(Q) \leqslant 0, n=1,2, \ldots, N\right\}=\varnothing$, либо если инфимум не достигается.

Введем функцию Лагранжа на $\widetilde{B} \times \mathbf{R}_{+}^{N}$ :

$$
L\left(Q, Y^{\prime}\right) \triangleq \mathbf{R}(Q)+\sum_{n=1}^{N} y_{n}^{\prime} \mathbf{S}_{n}(Q),
$$

где $0 \times \ll \pm \infty 》 \triangleq 0, \mathbf{R}_{+}^{N}=\left\{\left(y_{1}^{\prime}, \ldots, y_{N}^{\prime}\right): y_{1}^{\prime} \geqslant 0, \ldots, y_{N}^{\prime} \geqslant 0\right\}$.

Условие 1. Функиия $L(Q, 1)$ ограничена снизу на $\widetilde{B} ;$ существует такая точка $\widehat{Q} \in \widetilde{B}$, что $-\infty<\mathrm{S}_{n}(\widehat{Q})<0, n=1,2, \ldots, N,-\infty<$ $\mathbf{R}(\widehat{Q})<+\infty$.

Удалим из множества $\widetilde{B}$ все точки $Q$, в которых $\mathbf{R}(Q)= \pm \infty$, либо $\mathbf{S}_{n}(Q)= \pm \infty$ для некоторого $n$. Очевидно, при этом решение задачи (14) не изменится, множество $\widetilde{B}$ останется выпуклым, а все функционалы $\mathbf{R}, \mathrm{S}_{n}, n=1,2, \ldots, N$ будут линейными. В дальнейшем считаем, что условие 1 выполнено и множество $\widetilde{B}$ скорректировано. При этом к задаче (14) применимы все общие результаты теории выпуклого программирования [20].

$\mathrm{O}$ п р е д е л е н и е. Ограничение с номером $n$ назовем существенным, если при его отбрасывании ( $N$ уменьшается на единищу) решение задачи дает строго меньшее значение критерия $\mathbf{R}(Q)$.

Предположим, что как исходная задача, так и задача с отброшенным $n$-м ограничением имеют решение (инфимум достигается). Легко показать, что при этом ограничение с номером $n$ существенно тогда и только тогда, когда при его отбрасывании получается такая задача, что для всякого ее решения $Q^{*}$ справедливо строгое неравенство $\mathrm{S}_{n}\left(Q^{*}\right)>0$.

О п р е д е л е н и е. 1) $g\left(Y^{\prime}\right) \triangleq \inf _{Q \in \widetilde{B}} L\left(Q, Y^{\prime}\right)$ при $Y^{\prime} \in \mathbf{R}_{+}^{N}$; если $Y^{\prime} \in \mathbf{R}^{N} \backslash \mathbf{R}_{+}^{N}$, то $g\left(Y^{\prime}\right) \triangleq-\infty$.

2) Функцией возмущения называется функщия $\phi(Y) \triangleq \inf \{\mathbf{R}(Q): Q \in$ $\left.\widetilde{B}, \mathbf{S}_{n}(Q) \leqslant y_{n}, n=1,2, \ldots, N\right\}$ на $\mathbf{R}^{N}$.

Очевидно, функция $g$ вогнута, а функция возмушения $\phi$ выпукла; кроме того, $\phi(\mathbf{0})=\inf _{Q \in \widetilde{B}} \sup _{Y^{\prime} \in \mathbf{R}_{+}^{N}} L\left(Q, Y^{\prime}\right)$.

Теорема 1. Пусть выполнено условие 1. Тогда справедливы следующие утверждения. 
1) Функчия возмущения $\phi$ непрерьвна в нуле и субдифференчируема в нуле; вектор $Y_{*}^{\prime}$ обладает свойствами $\phi(0)=g\left(Y_{*}^{\prime}\right), Y_{*}^{\prime} \in \mathbf{R}_{+}^{N}$, тогда $u$ mолько тогда, когда $-Y_{*}^{\prime} \in \partial \phi(0)$;

2) справедливо соотношение дөойственности:

$$
\inf _{Q \in \widetilde{B}} \sup _{Y^{\prime} \in \mathbf{R}_{+}^{N}} L\left(Q, Y^{\prime}\right)=\sup _{Y^{\prime} \in \mathbf{R}_{+}^{N}} \inf _{Q \in \widetilde{B}} L\left(Q, Y^{\prime}\right)
$$

3) точка $Q^{*} \in \widetilde{B}$ является решением задачи (14) тогда и только тогда, когда найдется вектор $Y_{*}^{\prime} \in \mathbf{R}_{+}^{N}$, для которого өыполнено одно из следующих дөух эквивалентных утверждений:

а) пара $\left(Q^{*}, Y_{*}^{\prime}\right)$ является седловой точкой функции Лагранжа

$$
L\left(Q^{*}, Y^{\prime}\right) \leqslant L\left(Q^{*}, Y_{*}^{\prime}\right) \leqslant L\left(Q, Y_{*}^{\prime}\right)
$$

nрu всех $\left(Q, Y^{\prime}\right) \in \widetilde{B} \times \mathbf{R}_{+}^{N}$;

б) $\mathbf{S}_{n}\left(Q^{*}\right) \leqslant 0, n=1,2, \ldots, N ; L\left(Q^{*}, Y_{*}^{\prime}\right)=\min _{Q \in \widetilde{B}} L\left(Q, Y_{*}^{\prime}\right)$ u вblполнено «условие дополняющей нежесткости» $\sum_{n=1}^{N} y_{n *}^{\prime} S_{n}\left(Q^{*}\right)=0$.

Вектор $Y_{*}^{\prime} \in \mathbf{R}^{N}$ обладает указанными свойствами тогда и только mozda, когдa $-Y_{*}^{\prime} \in \partial \phi(\mathbf{0})$.

4) $-Y_{*}^{\prime} \in \partial \phi(\mathbf{0})$ mогда и только тогда, когда $Y_{*}^{\prime} \in \mathbf{R}_{+}^{N}-$ точка, для которой $g\left(Y_{*}^{\prime}\right)=\sup _{Y^{\prime} \in \mathbf{R}_{+}^{N}} g\left(Y^{\prime}\right)$.

Д о к а з а т е л ь с т в о. Пункты 1), 2) следуют непосредственно из теорем $0.33-0.35[20]$, п. 3) 一 из общеизвестных критериев оптимальности [20]. Для доказательства пा. 4) воспользуемся пп. 1), 2):

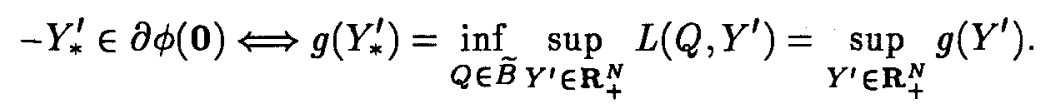

Следствие 2. Пусть выполнено условие 1, задача (14) имеет решение и ограничение с номером п существенно. Предположим, ито для задачи с отброшенным $n$-м ограничением также выполнено условие 1 , а коррекция множества $\widetilde{B}$ приводит $x$ тому же результату, ито и 6 исходной задаче. Тогда $y_{n *}^{\prime}>0$, где $-Y_{*}^{\prime} \in \partial \phi(0)$.

Д о к а з а т е л ь с т о. Пусть $Q^{*}$ - решение исходной задачи. Предположим, что $y_{n *}^{\prime}=0$ и отбросим $n$-е ограничение. Пусть $\widetilde{Y}_{*}^{\prime}-$ вектор, полученный из $Y_{*}^{\prime}$ отбрасыванием $n$-й компоненты. Тогда в новой задаче для $\left(Q^{*}, \widetilde{Y}_{*}^{\prime}\right)$ выполнен п. 26$)$ теоремы 1 , т.е. $Q^{*}$ - ее решение, что противоречит определению существенного ограничения.

\section{5. Алгоритм построения решения}

Начиная с данного раздела рассматривается однородная марковская модель, в которой

$$
\begin{aligned}
\lambda\left(\Gamma^{X} \mid a, \omega, t\right) & =\lambda\left(\Gamma^{X} \mid a, \xi_{t-}(\omega)\right), \quad R(\omega, t, a)=r\left(\xi_{t-}(\omega), a\right), \\
S_{n}(\omega, t, a) & =s_{n}\left(\xi_{t-}(\omega), a\right),
\end{aligned}
$$


$n=1,2, \ldots, N$. Без ограничений $(N=0)$ подобные дисконтированные модели изучались в [2], [8].

Условие 2. Пространство $X$ конеथно, топология $\tau^{X}$ дискретна; Функчи $r(x, a), s_{n}(x, a), n=1,2, \ldots, N$, равномерно ограниченны, $n p u-$ чем функчии $s_{n}$ мепрерывны, а функиия $r$ полунепрерывна снизу; пространство $\left(A, \tau^{A}\right)$ - компакт; функчия $\lambda(y \mid a, x)$ непрерывна.

Напомним некоторые факты относительно задач без ограничений $(N=0)$, при этом функцию штрафа удобно обозначить $V(x, a)$. Условие 2 считается выполненным, функция $V$ полагается полунепрерывной снизу и ограниченной.

Уравнение Беллмана

$$
\beta v(x)=\inf _{a \in A}\left\{V(x, a)-\lambda(X \mid a, x) v(x)+\sum_{y \in X \backslash x} v(y) \lambda(y \mid a, x)\right\}
$$

имеет единственное решение. Функция Беллмана имеет вид $e^{-\beta t} v(x)$. Существует оптимальный стационарный марковский селектор $\varphi^{*}\left(\xi_{t-}\right)$.

Следуюшие утверждения эквивалентны:

1) почти всюду по мере $Q^{\pi^{*}}$ выполнено равенство

$$
\begin{aligned}
& V\left(\xi_{t}, a\right)-\lambda\left(X \mid a, \xi_{t}\right) v\left(\xi_{t}\right)+\sum_{y \in X \backslash \xi_{t}} v(y) \lambda\left(y \mid a, \xi_{t}\right)=\beta v\left(\xi_{t}\right) \\
& \begin{aligned}
\mathrm{V}\left(Q^{\pi^{*}}\right) & \triangleq E^{\pi^{*}}\left[\int_{0}^{\infty} \int_{A} V\left(\xi_{t}, a\right) \pi^{*}(d a \mid \omega, t) e^{-\beta t} d t\right] \\
& =\sum_{x \in X} v(x) P_{0}(x)
\end{aligned}
\end{aligned}
$$

3) стратегия $\pi^{*}$ оптимальна.

Разрешимость уравнения (17) доказывается методом сжимаюших отображений. Для доказательства эквивалентности утверждений 1)-3) может быть полезным следующее представление:

$$
\begin{aligned}
E^{\pi}\left[\int_{t}^{\infty} \int_{A} V\left(\xi_{\theta_{-}}, a\right) \pi(d a \mid \omega, \theta) e^{-\beta \theta} d \theta \mid \mathcal{F}_{t}\right]=e^{-\beta t} v\left(\xi_{t}\right) \\
+E^{\pi}\left[\int _ { t } ^ { \infty } \left\{-\beta \vec{v}\left(\xi_{\theta_{-}}\right)+\int_{A}\left\{V\left(\xi_{\theta_{-}}, a\right)-v\left(\xi_{\theta-}\right) \lambda\left(X \mid a, \xi_{\theta_{-}}\right)\right.\right.\right. \\
\left.\left.\left.\quad+\sum_{y \in X \backslash \xi_{\theta_{-}}} v(y) \lambda\left(y \mid a, \xi_{\theta_{-}}\right)\right\} \pi(d a \mid \omega, \theta)\right\} e^{-\beta \theta} d \theta \mid \mathcal{F}_{t}\right]
\end{aligned}
$$

справедливое для любой ограниченной измеримой функции $v$ (в частности, для решения уравнения (17)).

Подробное изложение теории управляемых скачкообразных марковских процессов (без ограничений) можно найти в [1], [2], [4], [5], [7], при- 
чем, за исключением статьи [5], авторы сразу ограничиваются классом селекторов.

Очевидно, все аналогичные утверждения имеют место и для задачи максимизации дохода.

Переходим к описанию алгоритма решения задачи (14), считая выполненными условия 1,2 . По существу, этот алгоритм позволяет построить седловую точку функции Лагранжа в задаче выпуклого программирования с линейными ограничениями в произвольном линейном топологическом пространстве. В абстрактном виде описание этого алгоритма содержится в [21].

А1. Для каждого $Y^{\prime} \in \mathbf{R}_{+}^{N}$ значение $g\left(Y^{\prime}\right)$ определяется с помощью изложенного выше метода динамического программирования: $g\left(Y^{\prime}\right)=$ $\sum_{x \in X} v(x) P_{0}(x)$, где $v$ - решение (17) при

$$
V(x, a)=r(x, a)+\sum_{n=1}^{N} y_{n}^{\prime} s_{n}(x, a)
$$

А2. Для построения $-Y_{*}^{\prime} \in \partial \phi(0)$ в соответствии с п. 4) теоремы 1 следует максимизировать $g\left(Y^{\prime}\right)$ по $Y^{\prime} \in \mathbf{R}_{+}^{N}$, причем эта задача разрешима в силу п. 1) теоремы 1 . Для поиска $Y_{*}^{\prime}$ можно воспользоваться любым численным методом максимизации вогнутых функций.

При построении оптимальной стратегии воспользуемся п. 36) теоремы 1. Введем обозначения:

$$
V^{*}(x, a) \triangleq r(x, a)+\sum_{n=1}^{N} y_{* n}^{\prime} s_{n}(x, a)
$$

$v^{*}(x)$ - решение соответствуюшего уравнения Беллмана (17);

$$
D^{*} \triangleq\left\{(x, a): W(x, a)=\inf _{\tilde{a} \in A} W(x, \tilde{a})\right\},
$$

гце

$$
W(x, a) \triangleq V^{*}(x, a)+[K-\lambda(X \mid a, x)] v^{*}(x)+\sum_{y \in X \backslash x} v^{*}(y) \lambda(y \mid a, x),
$$

$K$ - константа, ограничивающая интенсивность выхода $\lambda(X \mid a, x)$. Символом $\widetilde{D}^{*}$ обозначим множество $\widetilde{D}^{*} \triangleq\left\{(\omega, t, a):\left(\xi_{t}(\omega), a\right) \in D^{*}\right\}$. Так как функция $W(x, a)$ полунепрерывна снизу, а пространство $X$ конечно, множество $D^{*}$ замкнуто; кроме того, существует такой стационарный селектор $\varphi$, что $\forall x \in X(x, \varphi(x)) \in D^{*}$. Согласно п. 3б) теоремы 1 , если $\pi^{*}$ - оптимальная стратегия, то соответствующая мера $Q^{\pi^{*}}$ сосредоточена на $\widetilde{D}^{*}$. Пусть $\Pi^{*}$ - множество всех стратегий $\pi$, для которых мера $Q^{\pi}$ сосредоточена на $\widetilde{D}^{*} ; B^{*} \triangleq\left\{Q^{\pi} \in \widetilde{B}, \pi \in \Pi^{*}\right\}$ - выпуклое подмножество $\widetilde{B}$. Ясно, что $\Pi^{*} \neq \varnothing ; B^{*} \neq \varnothing$. 
Очевидно, решение любой задачи вида

$$
\mathbf{V}(Q) \rightarrow \min , \quad Q \in B^{*},
$$

можно получить, решая безусловную задачу оптимизации

$$
\tilde{\mathbf{V}}(Q) \rightarrow \min
$$

в классе $Q \in \widetilde{B}$, где

$$
\widetilde{V}(x, a) \triangleq \begin{cases}V(x, a) & \text { при }(x, a) \in D^{*}, \\ C & \text { при }(x, a) \notin D^{*} ;\end{cases}
$$

- скорректированная функщия штрафа; $C$ - достаточно большая константа. Здесь $V$ - некоторая полунепрерывная снизу, ограниченная функция; функция $\tilde{V}$ обладает теми же свойствами в силу замкнутости $D^{*}$. Таким образом, считаем, что известен алгоритм решения оптимизационных задач без ограничений в классе $Q \in B^{*}$.

3 а м е ч а н и е. Обозначение $\mathbf{F}(Q)$ введено в разделе 2 для функций $F(\widetilde{\omega})$. Здесь и ниже имеется в виду, что в функцию $V(x, a)$ подставляется $\xi_{t-}(\omega)$ вместо $x$.

Если $N=0$, то в качестве $Q^{*}$ можно взять любой элемент $B^{*}$. Иначе говоря, все стратегии $\pi \in \Pi^{*}$ оптимальны.

Предположим, что имеется конструктивный метод решения исходной задачи оптимального управления с $(N-1)$-м ограничением, и построим соответствующий алгоритм для $N$ ограничений. Положим $\mathcal{A}_{1} \triangleq$ $\left\{n: y_{* n}^{\prime}>0\right\} ; \mathcal{A}_{2} \triangleq \mathcal{A} \backslash \mathcal{A}_{1} ; \mathcal{A}_{2}^{\prime}=\varnothing$.

А3. Если $\mathcal{A}_{2} \backslash \mathcal{A}_{2}^{\prime}=\varnothing$, то перейти к шагу А6.

А4. Выбрать произвольное $m \in \mathcal{A}_{2} \backslash \mathcal{A}_{2}^{\prime}$ и решить задачу $\mathbf{S}_{m}(Q) \rightarrow$ $\min$ в классе $Q \in B^{*}$ при ограничениях $\mathbf{S}_{n}(Q) \leqslant 0, n \in \mathcal{A}_{2}^{\prime}$.

Если минимум $\begin{cases}>0, & \text { то задача }(14) \text { решений не имеет. Конец. } \\ =0, & \text { то индекс } m \text { переводится из } \mathcal{A}_{2} \text { в } \mathcal{A}_{1} . \\ & \text { Перейти к шагу А3. } \\ <0, & \text { то перейти к шагу А5. }\end{cases}$

A5. $\mathcal{A}_{2}^{\prime}:=\mathcal{A}_{2}^{\prime} \cup\{m\}$, перейти к шагу А3.

A6. Если $\mathcal{A}_{1}=\varnothing$, то последняя из построенных точек $Q^{*}$ является искомой; конец. В противном случае выполнить шаг $\mathrm{A} 7$.

А7. Для всех подмножеств $\mathcal{A}_{1}^{+} \subseteq \mathcal{A}_{1}$ (включая $\mathcal{A}_{1}$ и $\varnothing$ ) выполнить шаги А8-A11.

А8. Положить $\mathcal{A}_{1}^{-} \triangleq \mathcal{A}_{1} \backslash \mathcal{A}_{1}^{+}, \mathcal{A}_{3}=\varnothing$ и ввести функции

$$
\widetilde{S}_{n}(\widetilde{\omega})= \begin{cases}-S_{n}(\widetilde{\omega}), & n \in \mathcal{A}_{1}^{+} \\ S_{n}(\widetilde{\omega}), & n \in \mathcal{A}_{1}^{-}\end{cases}
$$


A9. Решить задачу $\sum_{n \in \mathcal{A}_{1} \backslash \mathcal{A}_{3}} \tilde{\mathbf{S}}_{n}(Q) \rightarrow \min$ в классе $Q \in \dot{B}^{*}$ при ограничениях $\mathbf{S}_{m}(Q) \leqslant 0, m \in \mathcal{A}_{2}, \widetilde{\mathbf{S}}_{m}(Q) \leqslant 0, m \in \mathcal{A}_{3}$. Оптимальная точка в этой задаче обозначается $\widetilde{Q}$.

A10. Проверить условия:

Если $\left|\mathcal{A}_{1} \backslash \mathcal{A}_{3}\right|=1$ и построенный

минимум $\begin{cases}\leqslant 0, & \text { то закончить выполнение шагов А8-А11. } \\ >0, & \text { то задача (14) решений не имеет. Конец. }\end{cases}$

Если $\widetilde{\mathbf{S}}_{n}(\widetilde{Q})=0$ при всех $n \in \mathcal{A}_{1} \backslash \mathcal{A}_{3}$, то закончить выполнение шагов A8-A11.

Если $\widetilde{\mathbf{S}}_{n}(\widetilde{Q}) \geqslant 0$ для всех $n \in \mathcal{A}_{1} \backslash \mathcal{A}_{3}$, причем хотя бы одно неравенство строгое, то задача (14) решений не имеет. Конец.

А11. Выбрать индекс $n \in \mathcal{A}_{1} \backslash \mathcal{A}_{3}$, для которого $\widetilde{\mathbf{S}}_{n}(\widetilde{Q})<0$, включить его в множество $\mathcal{A}_{3}$ и перейти к шагу А9.

A12. В результате успешного выполнения шагов А8-A11 для всех $\mathcal{A}_{1}^{+} \subseteq \mathcal{A}_{1}$ в нашем распоряжении будет набор точек $\left.\widetilde{Q}_{k}, k=1,2, \ldots, 2^{\left|\mathcal{A}_{1}\right|}\right)$, для которых симплекс-методом линейного программирования следует определить коэффициенты $\lambda_{k}^{*} \geqslant 0, \sum_{k=1}^{2^{\left|\mathcal{A}_{1}\right|}} \lambda_{k}^{*}=1$, такие, что

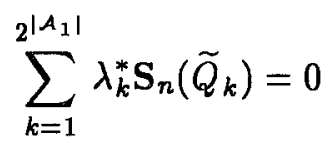

одновременно для всех $n \in \mathcal{A}_{1}$.

Построенная в результате точка $Q^{*}=\sum_{k=1}^{2^{\left|\mathcal{A}_{1}\right|}} \lambda_{k}^{*} \widetilde{Q}_{k}$ является решением задачи (14) в силу п. 3б) теоремы 1.

3 а м е ч а н ие. При выполнении шагов А4, А9 требование $Q \in$ $B^{*}$ обеспечивается путем указанной выше коррекции главной функции штрафа $s_{m}, \sum_{n \in \mathcal{A}_{1} \backslash \mathcal{A}_{3}} \tilde{s}_{n}$ соответственно. В результате получается задача с меньшим числом ограничений, для которой выполнены условия 1 , 2 , и можно применить изложенный алгоритм. Очевидно, в конечном счете все сводится к решению некоторого количества задач без ограничений с полунепрерывной снизу ограниченной функцией штрафа, а решением исходной задачи (14) является конечная выпуклая комбинация мер, отвечающих указанной серии задач без ограничений (эти меры порождаются стационарными марковскими селекторами). Изложенный алгоритм чрезвычайно трудоемок и, видимо, практически не применим для $N>3$. Поэтому его следует рассматривать лишь как доказательство принципиальной алгоритмической разрешимости проблемы. Кроме того, он используется в дальнейшем при доказательстве существования оптимальной стратегии.

В следующем разделе будет доказана разрешимость задачи (14) при условиях 1, 2 (точнее, при условии 3). При этом будут разрешимы все задачи с меньшим числом ограничений, возникающие на шагах А4, A9. 
Следовательно, при выполнении условия 3 изложенный алгоритм обязательно приведет к цели (это можно доказать с помощью индукции по $N$ ); варианты, приводяшие к выводу «задача (14) не имеет решений», исключены.

Если условие 3 не выполнено, то алгоритм может не дать ответа, даже если решение существует: задачи пунктов $\mathrm{A} 4$, А9 могут не решаться разработанными методами. В случае же успешного завершения работы алгоритма будет построена точка $Q^{*}$, решающая задачу (14) независимо от справедливости условий $1,2,3$. Действительно, во-первых, $\mathbf{S}_{n}\left(Q^{*}\right) \leqslant 0, n=1,2, \ldots, N$, и во-вторых, если для $Q \in \widetilde{B}$ выполнены эти неравенства, то $\mathbf{R}(Q) \geqslant L\left(Q, Y_{*}^{\prime}\right) \geqslant L\left(Q^{*}, Y_{*}^{\prime}\right)=\mathbf{R}\left(Q^{*}\right)$.

\section{6. Существование и свойства оптимальных стратегий}

Ограничимся временно классом стационарных марковских стратегий вида $\pi(\cdot \mid \omega, t)=\pi^{\mathrm{M}}\left(\cdot \mid \xi_{t-}(\omega)\right)$, где $\pi^{\mathrm{M}}(\cdot \mid x)$ - измеримое стохастическое ядро. Очевидно, всякое такое ядро действительно задает стратегию. Класс таких стратегий обозначим $\widetilde{\widetilde{\Pi}}^{\mathrm{M}}, \Pi^{\mathrm{M}}$ - класс стратегий $\pi$, для которых $Q^{\pi}$ есть конечная выпуклая комбинация мер $Q^{\widetilde{\pi}_{i}}$, где $\tilde{\tilde{\pi}}_{i} \in \widetilde{\widetilde{\Pi}}^{\mathrm{M}}$. Ясно, что $\widetilde{B}^{\mathrm{M}} \triangleq\left\{Q^{\pi}, \pi \in \Pi^{\mathrm{M}}\right\}$ есть множество всех конечных выпуклых комбинаций мер из $\widetilde{\widetilde{B}}^{\mathrm{M}} \triangleq\left\{Q^{\tilde{\tilde{\pi}}} \widetilde{\widetilde{\pi}}_{\tilde{B}} \widetilde{\widetilde{\Pi}}^{\mathrm{M}}\right\}$. В соответствии с леммой $1 \widetilde{B}^{\mathrm{M}}$ - выпуклое подмножество $\widetilde{B}$.

Рассмотрим задачу построения оптимальной в классе $\Pi^{\mathrm{M}}$ стратегии $\pi^{\mathrm{M}^{*}}: \mathbf{R}\left(Q^{\pi^{\mathrm{M}^{*}}}\right)=\inf _{\pi^{\mathrm{M}} \in \Pi^{\mathrm{M}} \cap \Pi} \mathbf{R}\left(Q^{\pi^{\mathrm{M}}}\right)$. Как и в разделе 4 , она сводится K задаче выпуклого программирования:

$\left.\begin{array}{l}\text { найти точку, дағщую инфимум функция } \mathbf{R}(Q) \text { на } \widetilde{B}^{\text {M }} \\ \text { при ограничениях } \mathbf{S}_{n}(Q) \leqslant 0, n=1,2, \ldots, N .\end{array}\right\}$

Условие 3. Выполнены условия 1,2 , причем почка $\widehat{Q}$ из условия 1 лежит $6 \widetilde{B}^{\mathrm{M}}$.

Очевидно, при этих условиях для задачи (21) справедливы все результаты, полученные в разделах 4,5 .

Теорема 2. Если выполнено условие 3 , то задача (21) имеет реuение.

Доказательству предпошлем некоторые вспомогательные построения и рассуждения. Пусть $\eta\left(\Gamma^{X}\right)=\sum_{x \in X} I\left\{x \in \Gamma^{X}\right\} /|X|$ - равномерная стохастическая мера на $X$. Для всякого стохастического ядра $\pi^{\mathrm{M}}(\mathrm{da} \mid x)$ обозначим $\widehat{P}^{\pi^{\mathrm{M}}}(d x \times d a)=\eta(d x) \pi^{\mathrm{M}}(d a \mid x) ; \widehat{B}-$ множество всех мер $\widehat{P}^{\pi^{\mathrm{M}}}$ на $X \times A$ указанного вида. На множествах $\widehat{B}, P(X \times A)$ (всевозможные стохастические меры на $X \times A)$ зафиксируем слабую топологию. Как известно [22], при этом $P(X \times A)$ - метризуемый компакт; замкнутость 
$\widehat{B} \subseteq P(X \times A)$ достаточно очевидна. Так что $\widehat{B}-$ тоже метризуемый компакт.

Пусть $\widehat{P}^{\pi^{\mathrm{M}}} \in \widehat{B}$ и $V(x, a)$ - произвольная измеримая функция на $X \times A$. Символом $\mathcal{V}\left(\widehat{P}^{\pi^{\mathrm{M}}}\right)$ обозначим величину

$$
\mathcal{V}\left(\widehat{P}^{\pi^{\mathrm{M}}}\right) \triangleq \mathrm{V}\left(Q^{\pi^{\mathrm{M}}}\right) .
$$

Здесь стратегия, отвечаюшая ядру $\pi^{\mathrm{M}}$, обозначается тем же символом. Формула (22) задает однозначное отображение из $\widehat{B}$ на расширенную прямую. Действительно, если $\widehat{P}^{\pi_{1}^{\mathrm{M}}}=\widehat{P}^{\pi_{2}^{\mathrm{M}}}$, то $\pi_{1}^{\mathrm{M}}(\cdot \mid x)=\pi_{2}^{\mathrm{M}}(\cdot \mid x)$ для любого $x \in X$, откуда получаем: $P^{\pi_{1}^{\mathrm{M}}}=P^{\pi_{2}^{\mathrm{M}}} \Longrightarrow Q^{\pi_{1}^{\mathrm{M}}}=Q^{\pi_{2}^{\mathrm{M}}}$.

Лемма 3. Пусть выполнено условие 3 и функиия $V(x, a)$ непрерывна. Тогда отображение (22) непрерывно.

Д о к а 3 а т е л в с т в о. Пусть $\pi^{\mathrm{M}}(\cdot \mid x)$ - стохастическое ядро на $A$. Рассмотрим уравнение

$$
G \circ F^{*}(x)=F^{*}(x),
$$

где $G$ - оператор, действуюший в пространстве действительных функций на $X$ с равномерной нормой $\rho$ :

$$
\begin{aligned}
G \circ F(x)=\frac{1}{\beta+K} \int_{A}\{ & V(x, a)+[K-\lambda(X \mid a, x)] F(x) \\
& \left.+\sum_{y \in X \backslash x} F(y) \lambda(y \mid a, x)\right\} \pi^{\mathrm{M}}(d a \mid x) .
\end{aligned}
$$

Очевидно, $G$ - сжимающий оператор:

$$
\rho\left(G \circ F^{1}, G \circ F^{2}\right) \leqslant \frac{K}{\beta+K} \rho\left(F^{1}, F^{2}\right),
$$

и поэтому $G^{i} \circ 0(x) \longrightarrow F^{*}(x)$ при $i \rightarrow \infty$.

Используя представление (19), легко убедиться, что

$$
\mathcal{V}\left(\widehat{P}^{\pi^{\mathrm{M}}}\right)=\sum_{x \in X} F^{*}(x) P_{0}(x)
$$

Пусть $\widehat{P}^{\pi_{n}^{\mathrm{M}}} \longrightarrow \widehat{P}^{\pi^{\mathrm{M}}}$ при $n \rightarrow \infty$. Нам нужно показать, что

$$
\lim _{n \rightarrow \infty} \mathcal{V}\left(\widehat{P}^{\pi_{n}^{\mathrm{M}}}\right)=\mathcal{V}\left(\hat{P}^{\pi^{\mathrm{M}}}\right)
$$

Введем операторы $G\left(\pi_{n}^{\mathrm{M}}\right), G\left(\pi^{\mathrm{M}}\right)$, которые задаются соответствующими стохастическими ядрами по формуле (23). Зафиксируем произвольное $\widetilde{\varepsilon}>0$. Из непрерывности $V$ следует, что $\exists N_{0}(\widetilde{\varepsilon}): \forall n>N_{0}(\widetilde{\varepsilon})$ 
$\rho\left(G\left(\pi_{n}^{\mathrm{M}}\right) \circ 0, G\left(\pi^{\mathrm{M}}\right) \circ 0\right)<\widetilde{\varepsilon}$. Согласно $(24)$ получаем:

$$
\begin{aligned}
& \rho\left(G^{2}\left(\pi_{n}^{\mathrm{M}}\right) \circ 0, G^{2}\left(\pi^{\mathrm{M}}\right) \circ 0\right) \leqslant \rho\left(G\left(\pi_{n}^{\mathrm{M}}\right) \circ G\left(\pi_{n}^{\mathrm{M}}\right) \circ 0, G\left(\pi_{n}^{\mathrm{M}}\right) \circ G\left(\pi^{\mathrm{M}}\right) \circ 0\right) \\
& \quad+\rho\left(G\left(\pi_{n}^{\mathrm{M}}\right) \circ G\left(\pi^{\mathrm{M}}\right) \circ 0, G\left(\pi^{\mathrm{M}}\right) \circ G\left(\pi^{\mathrm{M}}\right) \circ 0\right) \\
& \leqslant \\
& \quad \frac{K}{\beta+K} \widetilde{\varepsilon}+\rho\left(G\left(\pi_{n}^{\mathrm{M}}\right) \circ F^{1}, G\left(\pi^{\mathrm{M}}\right) \circ F^{1}\right),
\end{aligned}
$$

где $F^{1} \triangleq G\left(\pi^{\mathrm{M}}\right) \circ 0$. Снова воспользуемся сходимостью $\widehat{P}^{\pi_{n}^{\mathrm{M}}} \longrightarrow \widehat{P}^{\mathrm{M}}$ при $n \rightarrow \infty: \exists N_{1}(\widetilde{\varepsilon}) \geqslant N_{0}(\widetilde{\varepsilon}): \forall n>N_{1}(\widetilde{\varepsilon}) \rho\left(G\left(\pi_{n}^{\mathrm{M}}\right) \circ F^{1}, G\left(\pi^{\mathrm{M}}\right) \circ F^{1}\right)<\widetilde{\varepsilon}$, т.e. $\rho\left(G^{2}\left(\pi_{n}^{\mathrm{M}}\right) \circ 0, G^{2}\left(\pi^{\mathrm{M}}\right) \circ 0\right)<[K /(\beta+K)+1] \widetilde{\varepsilon}$. Продолжая эти рассуждения дальше, убеждаемся, что и при любом $i=1,2, \ldots \exists N_{i}(\widetilde{\varepsilon}): \forall n>N_{i}(\widetilde{\varepsilon})$ $\rho\left(G^{i+1}\left(\pi_{n}^{\mathrm{M}}\right) \circ 0, G^{i+1}\left(\pi^{\mathrm{M}}\right) \circ 0\right)<\psi(i) \widetilde{\varepsilon}$, где $\psi(i)$ - некоторая функцाя с параметрами $K, \beta$.

Выберем произвольное $\varepsilon>0$ и возьмем $i$ из условий

$$
\rho\left(G^{i+1}\left(\pi_{n}^{\mathrm{M}}\right) \circ 0, F_{n}^{*}\right)<\frac{\varepsilon}{3}, \quad \rho\left(G^{i+1}\left(\pi^{\mathrm{M}}\right) \circ 0, F^{*}\right)<\frac{\varepsilon}{3} .
$$

Здесь $F_{n}^{*}, F^{*}$ - неподвижные точки операторов $G\left(\pi_{n}^{\mathrm{M}}\right), G\left(\pi^{\mathrm{M}}\right) ; i$ не зависит от $n$ в силу (24). Осталось положить $\widetilde{\varepsilon}=\varepsilon /(3 \psi(i))$ и убедиться, что $\rho\left(F_{n}^{*}, F^{*}\right)<\varepsilon$ для любого $n>N_{i}(\widetilde{\varepsilon})$. Для завершения доказательства следует воспользоваться формулой (25).

Д ок а з а т е л с с т в о т е о рем ы 2. Рассмотрим множество $\mathcal{N}$ всех стохастических мер на $\widehat{B}$. Очевидно, $\mathcal{N}-$ выпуклый метризуемый компакт (в слабой топологии) [22]. Введем функционал

$$
\tilde{\mathcal{V}}(\nu) \triangleq \int_{\widehat{B}} \mathcal{V}(\widehat{P}) \nu(d \widehat{P})
$$

где $\mathcal{V}$ - функционал (22) на $\widehat{B}$. В соответствии с леммой 3 функционал $\widetilde{\mathcal{V}}(\nu)$ непрерывен, если непрерывна задающая функция $V$, и полунепрерывен снизу и ограничен, если такова $V$. (Функция $V$ полунепрерывна и ограничена снизу тогда и только тогда, когда существует последовательность ограниченных непрерывных функций $V_{n} \uparrow V$ [22].)

Пусть $\widetilde{\mathcal{V}}_{r}$ задается функцией $r$, а $\widetilde{\mathcal{V}}_{s_{n}}$ - функциями $s_{n}, n=1,2, \ldots, N$. Ясно, что при условии 3 задача

$$
\left.\begin{array}{ll}
\widetilde{\mathcal{V}}_{r}(\nu) \rightarrow \min , & \nu \in \mathcal{N} \\
\widetilde{\mathcal{V}}_{s_{n}}(\nu) \leqslant 0, & n=1,2, \ldots, N
\end{array}\right\}
$$

имеет решение. Более того, для нее справедливы все результаты разделов 4,5 . В результате выполнения шагов $\mathrm{A} 1, \mathrm{~A} 2$ будет получен тот же вектор $Y_{*}^{\prime}$, что и для задачи (21), а также для задачи (14). Пусть $\mathcal{N}^{*}=\left\{\nu^{*}: L\left(\nu^{*}, Y_{*}^{\prime}\right)=\inf _{\nu \in \mathcal{N}} L\left(\nu, Y_{*}^{\prime}\right)\right\}$, где $L-$ функция Лагранжа для (27). Решениями задач без ограничений

$$
\tilde{\mathcal{V}}_{V}(\nu) \rightarrow \min , \quad \nu \in \mathcal{N}^{*}
$$


( $V$ - ограниченная полунепрерывная снизу функция, задающая функционалы $\mathcal{V}_{V}, \widetilde{\mathcal{V}}_{V}$ по формулам $(22),(26)$ ) будут стохастические меры, сосредоточенные на множестве таких $\widehat{P}^{\pi^{\mathrm{M}}} \in \widehat{B}$, для которых точки $Q^{\pi^{\mathrm{M}}}$ являются решениями соответствуюших задач (20). Поэтому решение задачи (28) можно построить, скорректировав целевую функцию так, как указано ранее, и решив соответствуюшую безусловную задачу- $\mathrm{B}$ результате получится марковский селектор $\varphi^{*}$, и в качестве решения задачи (28) следует взять меру Дирака, сосредоточенную в точке $\widehat{P}^{\varphi^{*}}$.

Теперь, рассуждая по индукции $(N=0,1,2, \ldots)$, убеждаемся, что для любого заданного $N$ алгоритм из раздела 5 , примененный к задаче (27), даст ее решение в виде конечной смеси мер Дирака, сосредоточенных в точках $\widehat{P}$, отвечающих стационарным марковским селекторам. Соответствуюшая выпуклая комбинация мер из $\widetilde{\widetilde{B}}^{\mathrm{M}}$ даст решение задачи (21) (здесь существенна конечность этой комбинации).

Следствие 3. Если выполнено условие 3, то исходная задача (14) имеет решение.

Д о к а з а т е л ь с т в о. Как уже отмечалось, на каждом шаге работы алгоритма (раздел 5) над задачами (21), (27) будут получаться конечные смеси стационарных марковских селекторов (этим не совсем строгим термином обозначены соответствующие точки в пространствах $\left.\widetilde{B}^{\mathrm{M}}, \mathcal{N}\right)$. Достаточно очевидно, что при работе алгоритма над задачей (14) будут получаться соответствуюшие конечные выпуклые комбинации мер $Q$ из $\widetilde{B}$, отвечаюших стационарным селекторам; в итоге мы получим точку $Q^{*} \in \widetilde{B}$, для которой выполнен п. 36) теоремы 1.

3 а м е ч а и е. Если выполнено условие 3, то все шаги алгоритма из раздела 5 над задачами (14), (21), (27) будут успешно выполнены: возникающие в пунктах A4, А9 задачи с меньшим числом ограничений успешно решаются с помошью этого же алгоритма, а варианты, приводящие к выводу «исходная задача не имеет решений», исключены.

Теорема 3. Если өыполнено условие 3 , то существуют стационарные марковские селекторы $\varphi_{j}, j=1,2, \ldots, N+1$ такие, ито некоторая выпуклая их комбинация (смесь)

$$
Q^{*}=\sum_{j=1}^{N+1} \lambda_{j} Q^{\varphi_{j}} \quad\left(\lambda_{j} \geqslant 0, \sum_{j=1}^{N+1} \lambda_{j}=1\right)
$$

является решением задачи (14).

Д ок азат е л ь с т в о. Пусть $\widetilde{Q}^{*}$ - некоторое решение задачи (14), существующее в силу следствия 3 . Как было установлено при его доказательстве, существует такой конечный набор стационарных мар- 
ковских селекторов $\varphi_{m}, m=1,2, \ldots, M$, что

$$
\widetilde{Q}^{*}=\sum_{m=1}^{M} z_{m}^{*} Q^{\varphi_{m}}
$$

где $z_{m}^{*} \geqslant 0, \sum_{m=1}^{M} z_{m}^{*}=1$. Кроме того, $Q^{\varphi_{m}} \in B^{*}$ для любого $m=$ $1,2, \ldots, M$.

Введем симплекс $\mathbf{Z}=\left\{Z \in \mathbf{R}^{M}: z_{m} \geqslant 0, \sum_{m=1}^{M} z_{m}=1\right\}$ и отображение $Q: \mathbf{Z} \rightarrow B^{*}$ по формуле $Q(Z)=\sum_{m=1}^{M} z_{m} Q^{\varphi_{m}}$. Ясно, что для всех $Z \in \mathbf{Z}$

$$
L\left(Q(Z), Y_{*}^{\prime}\right)=\min _{Q \in \widetilde{B}} L\left(Q, Y_{*}^{\prime}\right)
$$

Рассмотрим множество $G=\left\{\left\{\mathbf{S}_{n}(Q(Z))\right\}_{n=1}^{N}, Z \in \mathbf{Z}\right\} \subset \mathbf{R}^{N}$. Очевицно, $G$ ограниченно и выпукло. Далее, если $g^{k} \in G, \lim _{k \rightarrow \infty} g^{k}=g$, то из последовательности прообразов точек $g$ можно извлечь сходящуюся в компакте $\mathbf{Z}$ подпоследовательность $Z^{k_{i}} \longrightarrow Z \in \mathbf{Z},(i \rightarrow \infty)$ причем вследствие непрерывности отображения $\boldsymbol{g}(\cdot) \triangleq\left\{\mathbf{S}_{n}(Q(\cdot))\right\}_{n=1}^{N}$ имеем: $\lim _{i \rightarrow \infty} g^{k_{i}}=g=g(Z)$. То есть $G$ - замкнуто. Итак, $G$ - выпуклый компакт. По теореме Каратеодори [23] всякая точка из $G$ может быть представлена как выпуклая комбинация из $N+1$ крайней точки $G$; в частности, $g\left(Z^{*}\right)=\sum_{j=1}^{N+1} \lambda_{j} g_{j}$. Поскольку $g(\cdot)$ - непрерывное аффинное отображение из $\mathbf{Z}$ в $G$, для каждой крайней точки $g_{j}, j=1,2, \ldots, N+1$, найдется крайняя в $\mathbf{Z}$ точка $Z_{j}$ такая, что $g_{j}=g\left(Z_{j}\right)$ [24]. Ясно, что все векторы $Z_{j}$ являются векторами стандартного базиса в $\mathbf{R}^{M}$, которым соответствуют стационарные марковские селекторы $\varphi_{j}, j=1,2, \ldots, N+1$.

Докажем, что точка $Q^{*} \triangleq \sum_{j=1}^{N+1} \lambda_{j} Q^{\varphi_{j}}$ - искомая. Во-первых, $\left\{\mathbf{S}_{n}\left(Q^{*}\right)\right\}_{n=1}^{N}=g\left(Z^{*}\right)=\left\{\mathbf{S}_{n}\left(\widetilde{Q}^{*}\right)\right\} ;$ во-вторых, теперь из (29) следует равенство $\mathbf{R}\left(Q^{*}\right)=\mathbf{R}\left(\widetilde{Q}^{*}\right)$. Следовательно, $Q^{*}$, как и $\widetilde{Q}^{*}$, является решением задачи (14). Отметим, что точки $Q^{*}, \widetilde{Q}^{*}$ не обязаны совпадать.

\section{7. Пример}

Рассмотрим одноканальную марковскую систему массового обслуживания (СМО) с отказами. Положим $X=\{0,1\}$, где $x=0(x=1)$ означает, что СМО свободна (занята); $A=\{0,1\}$, где $a=0(a=1)$ означает, что применяется менее (более) интенсивный режим обслуживания; $r(x, a)=e_{a} ; \lambda(1 \mid a, 0)=\lambda ; \lambda(0 \mid a, 1)=\mu_{a} ; s(x, a)=-\beta d+x \lambda c$. Здесь $e_{0}\left(e_{1}\right)$ - скорость потерь, связанная с менее (более) интенсивным обслуживанием, $\lambda$ - интенсивность входного потока заявок, $\mu_{0}, \mu_{1}-$ интенсивности обслуживания при соответствующих управлениях, $c$ штраф за потерю заявки, $\beta$ - как обычно, коэффициент дисконтирования. Положительные числа $e_{0}<e_{1}, \lambda, \mu_{0}<\mu_{1}, \beta, d, c$ считаются заданными. 
Очевидно, для произвольной стратегии $\pi \mathbf{R}\left(Q^{\pi}\right)-$ полные дисконтированные потери на обслуживание, а $\mathbf{S}\left(Q^{\pi}\right)+d$ - полный дисконтированный штраф за потерю заявок. Таким образом, задача $\mathbf{R}\left(Q^{\pi}\right) \rightarrow \min$ при ограничении $\mathbf{S}\left(Q^{\pi}\right) \leqslant 0$ (рассматривается случай $N=1$, поэтому индекс $n$ всюду опускается) означает, что требуется минимизировать потери на обслуживание при условии, что штраф за потерю заявок не превосходит данной константы $d$. Вероятность свободного начального состояния СМО считаем равной $p_{0} ; \xi_{t}-$ состояние CMO в момент $t$.

В соответствии с разделом 5 введем функцию Лагранжа $L\left(Q^{\pi}, y^{\prime}\right)=$ $\mathbf{R}\left(Q^{\pi}\right)+y^{\prime} \mathbf{S}\left(Q^{\pi}\right)$ и решим безусловную задачу оптимизации $L\left(Q^{\pi}, y^{\prime}\right) \rightarrow$ min. Анализ уравнения Беллмана

$$
\beta v(x)=\min _{a \in A}\left\{e_{a}+y^{\prime}(x \lambda c-\beta d)+\left[(1-x) \lambda+x \mu_{a}\right][v(1-x)-v(x)]\right\}
$$

дает следуюшие результаты.

а) При $y^{\prime} \leqslant y_{*}^{\prime} \triangleq \frac{\left(e_{1}-e_{0}\right)\left(\beta+\lambda+\mu_{0}\right)}{\lambda c\left(\mu_{1}-\mu_{0}\right)}$

$$
\begin{aligned}
& v(0)=\frac{1}{\beta}\left[e_{0}+y^{\prime} \frac{\lambda^{2} c}{\beta+\lambda+\mu_{0}}\right]-y^{\prime} d \\
& v(1)=\frac{1}{\beta}\left[e_{0}+y^{\prime} \frac{\lambda^{2} c+\beta \lambda c}{\beta+\lambda+\mu_{0}}\right]-y^{\prime} d .
\end{aligned}
$$

б) При $y^{\prime}>y_{*}^{\prime}$

$$
\begin{aligned}
& v(0)=\frac{1}{\beta}\left[e_{0}+\lambda \frac{e_{1}-e_{0}}{\beta+\lambda+\mu_{1}}+y^{\prime} \frac{\lambda^{2} c}{\beta+\lambda+\mu_{1}}\right]-y^{\prime} d \\
& v(1)=\frac{1}{\beta}\left[e_{0}+\frac{(\lambda+\beta)\left(e_{1}-e_{0}\right)}{\beta+\lambda+\mu_{1}}+y^{\prime} \frac{\lambda^{2} c+\beta \lambda c}{\beta+\lambda+\mu_{1}}\right]-y^{\prime} d .
\end{aligned}
$$

в) Оптимальным является стационарный марковский селектор $\varphi^{*}(x)=x I\left\{y^{\prime}>y_{*}^{\prime}\right\}$; при $y^{\prime}=y_{*}^{\prime}$ оптимальны селекторы

$$
\varphi_{0}(x) \equiv 0, \quad \varphi_{1}(x)=x .
$$

г) $g\left(y^{\prime}\right)=\inf _{Q \in \widetilde{B}} L\left(Q, y^{\prime}\right)=p_{0} v(0)+\left(1-p_{0}\right) v(1)$.

Рассмотрим селекторы (30). Нетрудно убедиться, что

$$
\begin{aligned}
& \mathbf{R}\left(Q^{\varphi_{0}}\right)=\frac{e_{0}}{\beta} ; \quad \mathbf{R}\left(Q^{\varphi_{1}}\right)=\frac{1}{\beta}\left[e_{0}+\frac{\left(e_{1}-e_{0}\right)\left(\lambda+\beta\left(1-p_{0}\right)\right)}{\beta+\lambda+\mu_{1}}\right] \\
& \mathbf{S}\left(Q^{\varphi_{0}}\right)=\frac{\lambda^{2} c+\left(1-p_{0}\right) \beta \lambda c}{\beta\left(\beta+\lambda+\mu_{0}\right)}-d ; \quad \mathbf{S}\left(Q^{\varphi_{1}}\right)=\frac{\lambda^{2} c+\left(1-p_{0}\right) \beta \lambda c}{\beta\left(\beta+\lambda+\mu_{1}\right)}-d .
\end{aligned}
$$

В зависимости от значений параметров возможны следующие варианты.

5 Теория вероятностей и ее применения, № 1 
1. $\lambda^{2} c+\left(1-p_{0}\right) \beta \lambda c-\beta^{2} d-\beta \lambda d-\beta \mu_{0} d \leqslant 0$. При этом $\inf _{Q \in \widetilde{B}} \mathbf{R}(Q)$ достигается при $\varphi_{0}$, а $\mathbf{S}\left(Q^{\varphi_{0}}\right) \leqslant 0$, т.е. в рассматриваемом случае ограничение не существенно. Графическое представление результатов дано на рис. 1 ниже.
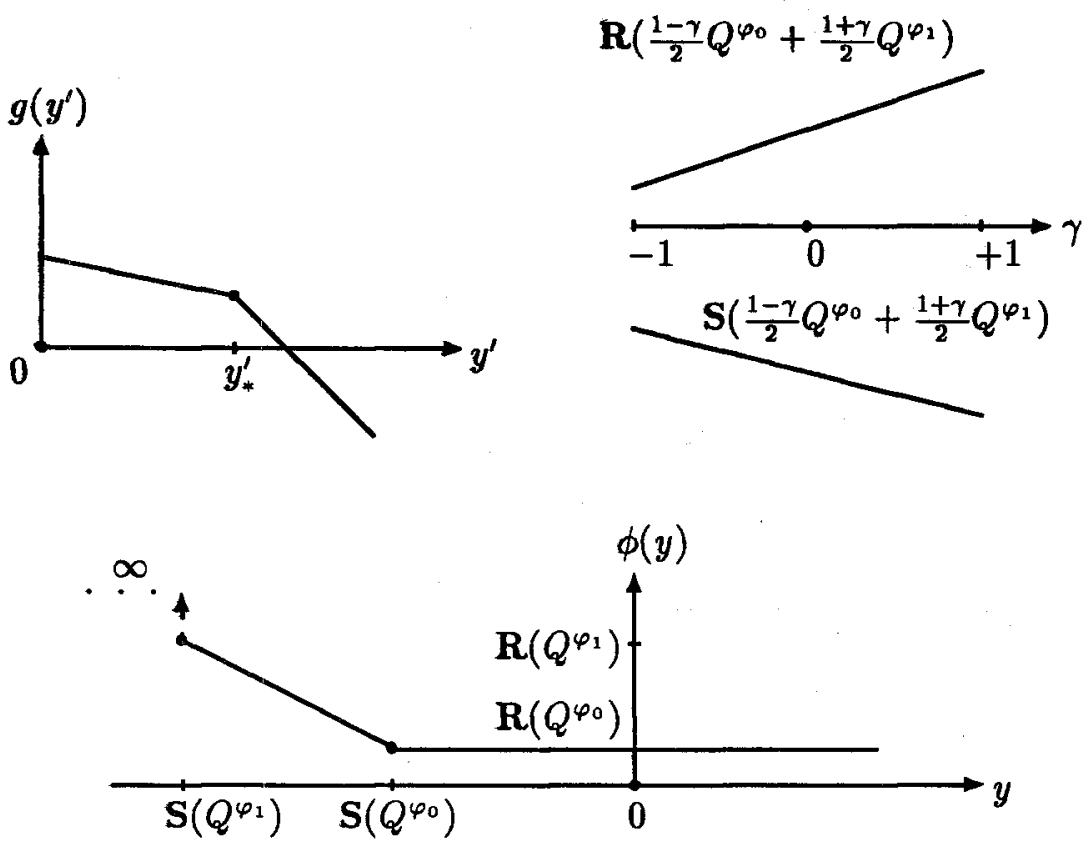

Puc. 1

2. $\lambda^{2} c+\left(1-p_{0}\right) \beta \lambda c-\beta^{2} d-\beta \lambda d-\beta \mu_{1} d>0$. При этом $\inf _{Q \in \widetilde{B}} \mathbf{S}(Q)=$ $\mathbf{S}\left(Q^{\varphi_{1}}\right)>0$ и в модели нет допустимых стратегий: $\Pi=\varnothing$. Графическое представление результатов дано на рис. 2.

3. $\lambda^{2} c+\left(1-p_{0}\right) \beta \lambda c-\beta^{2} d-\beta \lambda d-\beta \mu_{1} d \leqslant 0<\lambda^{2} c+\left(1-p_{0}\right) \beta \lambda c-\beta^{2} d-$ $\beta \lambda d-\beta \mu_{0} d$. Если здесь левое неравенство - строгое, то справедливы условия $1,2,3$; в случае равенства нарушено требование существования такой точки $\widehat{Q} \in \widetilde{B}$, что $\mathbf{S}(\widehat{Q})<0$. Графическое представление результатов дано на рис. 3.

Очевидно, в рассматриваемом случае

$$
\gamma^{*}=\frac{\mathbf{S}\left(Q^{\varphi_{0}}\right)+\mathbf{S}\left(Q^{\varphi_{1}}\right)}{\mathbf{S}\left(Q^{\varphi_{0}}\right)-\mathbf{S}\left(Q^{\varphi_{1}}\right)} \in(-1,1]
$$

и точка

$$
Q^{*}=0.5\left(1-\gamma^{*}\right) Q^{\varphi 0}+0.5\left(1+\gamma^{*}\right) Q^{\varphi_{1}}
$$

является рептением задачи (14). 

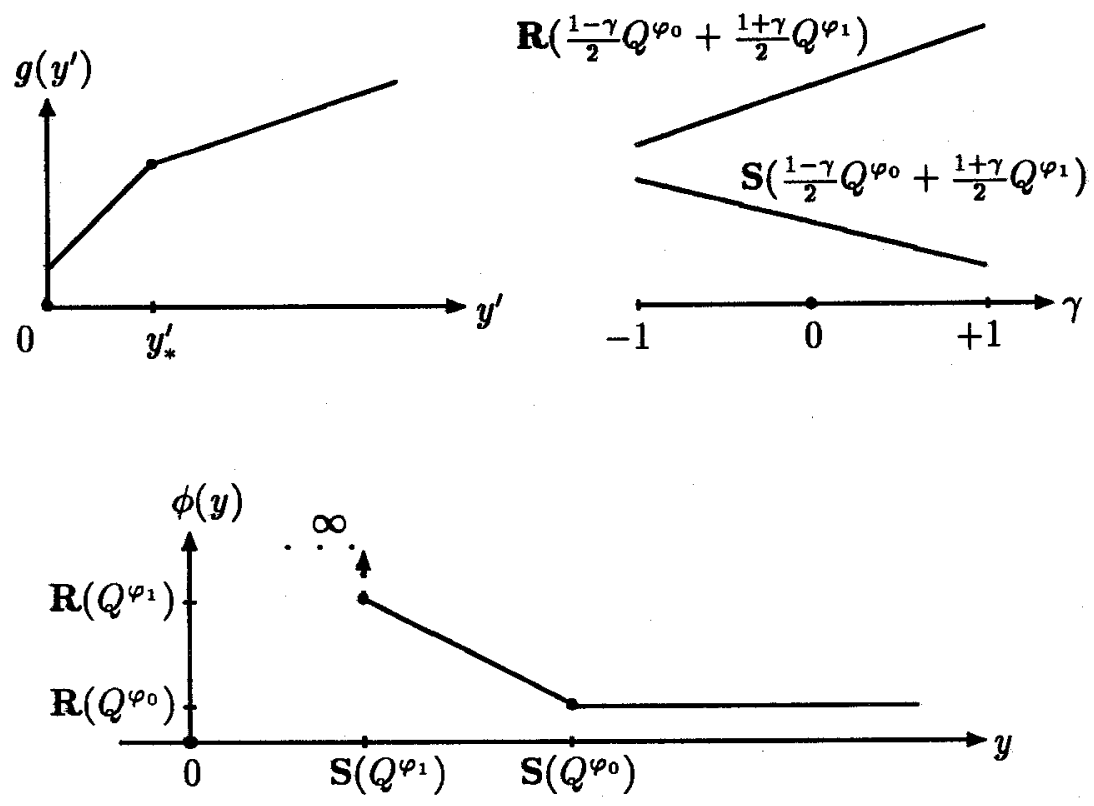

Pис. 2

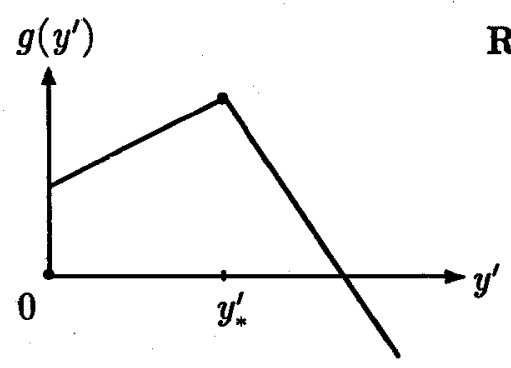

$\mathbf{R}\left(\frac{1-\gamma}{2} Q^{\varphi_{0}}+\frac{1+\gamma}{2} Q^{\varphi_{1}}\right)$

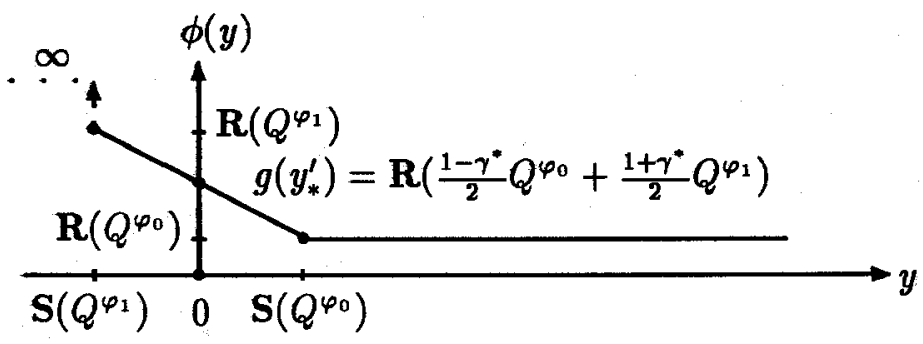

Pис. 3 
Ясно, что ограничиться классом стационарных селекторов нельзя; кроме того, параметр $\gamma^{*}$ оптимальной стратегии зависит от начального распределения. Существование оптимальной стратегии $\pi^{*}: Q^{\pi^{*}}=Q^{*}$ следует из леммы 1. Чтобы явно описать оптимальную стратегию, воспользуемся пп. 3б), 4 теоремы 1 . В соответствии с рис. $3 y_{*}^{\prime}>0$ - точка,

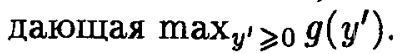

Ранее было установлено, что $\min _{\pi} L\left(Q^{\pi}, y_{*}^{\prime}\right)$ достигается при селек$\operatorname{topax}(30) ;$ более того, $L\left(Q^{\pi^{\mathrm{M}}}, y_{*}^{\prime}\right)=\min _{\pi} L\left(Q^{\pi}, y_{*}^{\prime}\right)$, если

$$
\pi^{\mathrm{M}}(0 \mid 0)=1, \quad \pi^{\mathrm{M}}(0 \mid 1)=\frac{1-\delta}{2}, \quad \pi^{\mathrm{M}}(1 \mid 1)=\frac{1+\delta}{2},
$$

где $\delta \in[-1,1]-$ произвольное фиксированное число. Легко убедиться, что

$$
\mathbf{S}\left(Q^{\pi^{\mathrm{M}}}\right)=\frac{\lambda^{2} c+\left(1-p_{0}\right) \beta \lambda c}{\beta(\beta+\lambda+\mu(\delta))}-d,
$$

где

$$
\mu(\delta) \triangleq 0.5(1-\delta) \mu_{0}+0.5(1+\delta) \mu_{1} .
$$

Теперь ясно, что оптимальное значение $\delta^{*}$ следует выбрать из условия $\mathbf{S}\left(Q^{\pi^{\mathrm{M}}}\right)=0$ :

$$
\mu\left(\delta^{*}\right)=\frac{\lambda^{2} c+\left(1-p_{0}\right) \beta \lambda c-\beta^{2} d-\beta \lambda d}{\beta d} .
$$

При этом рандомизированная марковская стратегия (31) будет оптимальной. Подчеркнем, что $\delta^{*}$ не обязано совпадать с $\gamma^{*}$.

В работах [16], [17] можно найти другие содержательные примеры синтеза оптимальных управлений в СМО с функциональными ограничениями.

\section{СПИСОК ЛИТЕРАТУРЫ}

1. Юикевич $A$. A. Управляемые марковские модели со счетным множеством состояний и непрерывным временем. - Теория вероятн. и ее примен., 1977, т. XXII, B. 2 , с. $222-241$.

2. Юuкевич $A . A$., Файнберг $E . A$. Об однородных управляемых марковских моделях с непрерывным временем и конечным или счетным множеством состояний. Теория вероятн. и ее примен., 1979, т. XXIV, в. 1, с. 155-160.

3. Boel R., Varaiya P. Optimal control of jump processes. - SIAM J. Control Optim., 1977 , v. 15 , № 1 , p. 92-119.

4. Юикевич A. А. Управляемые скачкообразные марковские модели. - Теория вероятн. и ее примен., 1980, т. XXV, в. 2, с. 247-270.

5. Китаев $М$. Ю. Полумарковские и скачкообразные марковские управляемые модели. - Теория вероятн. и ее примен., 1985, т. XXX, в. 2, с. 252-268.

6. Эллиотm P. Стохастический анализ и его приложения. М.: Мир, 1986.

7. Piunouski A. B., Khametov V. M. New effective solutions of optimality's equations for the controlled Markov chains with continuous parameter. - Problems of Control and Inform. Theory, 1985, v. 14, № 4, p. 303-318. 
8. Khametov V. M., Piunovski A. B. The optimal control of discounted Markov processes with infinite horizon. - In: II IFAC Symposium on Stochastic Control. Vilnius, USSR. 1986, part 1, p. 326-329.

9. Фрид $E$. Б. Об оптимальных стратегиях в задачах управления с ограничениями. - Теория вероятн. и ее примен., 1972, т. XVII, в. 1, с. 194-199.

10. Borkar V. S. Topics in Controlled Markov Chains. - Avon: Longman Scientific and Technical, England, 1991, v. 240.

11. Пиуновский $A$. Б. Управление случайными последовательностями в задачах с ограничениями. - Теория вероятн. и ее примен., 1993, т. 38, в. 4, с. 891-903.

12. Feinberg $E$. A., Shwartz A. Constrained discounted dynamic programming. - Math. Oper. Res., 1996, v. 21, p. 922-945.

13. Altman E. Denumerable constrained Markov decision processes and finite approximations. - Math. Oper. Res., 1994, v. 19, № 1, p. 169-191.

14. Пиуновский $A$. Б. Управление скачкообразными процессами в задачах с ограничениями. - Автоматика и телемеханика, 1994, № 4, с. 75-89.

15. Докучаев $H$. Г. Существование оптимальных управлений и необходимые услових оптимальности для частично наблюдаемых диффузионных процессов. - Дифференц. уравнения, 1994, т. 30, № 9, с. 1498-1507.

16. Feinberg E. A., Reiman M. I. Optimality of randomized trunk reservation. - Probab. Engin. Inform. Sci., 1994, v. 8, p. 463-489.

17. Feinberg E. A., Kim D. J. Bicriterion optimization of an $\mathrm{M} / \mathrm{G} / 1$ queue with a removable server. - Probab. Engin. Inform. Sci., 1996, v. 10, p. 57-73.

18. Липчер $P$. HI., IIиряев $A$. H. Теория мартингалов. М.: Наука, 1986, 512 с.

19. Jacod J. Multivariate point processes: predictable projection, Radon-Nicodym derivatives. - Z. Wahrscheinlichkeitstheor. verw. Geb., 1975, B. 31, H. 3, S. 235-253.

20. Левия В. Л. Выпуклый анализ. М.: Наука, 1985.

21. Пиуяовский $A$. Б. Задача выпуклого программирования с линейными ограничениями. - Журн. вычисл. матем. и матем. физики, 1994, т. 34, № 4, с. 545-553.

22. Бертсекас Д., ІІрив С. Стохастическое оптимальное управление. М.: Наука, $1985,280 \mathrm{c}$.

23. Схрейвер А. Теория линейного и целочисленного программирования. Т. 1. М.: Мир, 1971.

24. Мейер П.-А. Вероятность и потенциалы. М.: Мир, 1973, 334 с.

Поступила в редакцию 4.VII.1994 